\title{
Efficient identification of neoantigen- specific T-cell responses in advanced human ovarian cancer
}

Song Liu ${ }^{1{ }^{*}}$, Junko Matsuzaki ${ }^{2^{* \dagger}}$, Lei Wei ${ }^{1 \dagger}$, Takemasa Tsuji ${ }^{2 \dagger}$, Sebastiano Battaglia ${ }^{2 \dagger}$, Qiang Hu ${ }^{1}$, Eduardo Cortes ${ }^{1}$, Laiping Wong1', Li Yan', Mark Long1', Anthony Miliotto², Nicholas W. Bateman³, Shashikant B. Lele, Thinle Chodon ${ }^{2}$, Richard C. Koya ${ }^{2}$, Song Yao ${ }^{5}$, Qianqian Zhu', Thomas P. Conrads ${ }^{3,6,7}$, Jianmin Wang ${ }^{1}$, George L. Maxwell ${ }^{3,6}$, Amit A. Lugade 2 and Kunle Odunsi ${ }^{2,4^{*}}$

\begin{abstract}
Background: Efficient identification of neoantigen-specific T-cell responses in epithelial ovarian cancer (EOC) remains a challenge. Existing investigations of spontaneous T-cell response to tumor neoepitope in EOC have taken the approach of comprehensive screening all neoantigen candidates, with a validation rate of $0.5-2 \%$.

Methods: Whole-exome and transcriptome sequencing analysis of treatment-naive EOC patients were performed to identify neoantigen candidates, and the immunogenicity of prioritized neoantigens was evaluated by analyzing spontaneous neoantigen-specfic $\mathrm{CD}^{+}$and $\mathrm{CD} 8^{+}$T-cell responses in the tumor and/or peripheral blood. The biological relevance of neoantigen-specific T-cell lines and clones were analyzed by evaluating the capacity of autologous ovarian tumor recognition. Genetic transfer of T-cell receptor (TCR) from these neoantigen-specific T-cell clones into peripheral blood T-cells was conducted to generate neoepitope-specific T-cells. The molecular signature associated with positive neoantigen T-cell responses was investigated, and the impacts of expression level and lymphocyte source on neoantigen identification were explored.
\end{abstract}

Results: Using a small subset of prioritized neoantigen candidates, we were able to detect spontaneous $\mathrm{CD}^{+}$and/ or $\mathrm{CD} 8^{+}$T-cell responses against neoepitopes from autologous lymphocytes in half of treatment-naive EOC patients, with a significantly improved validation rate of 19\%. Tumors from patients exhibiting neoantigen-specific T-cell responses exhibited a signature of upregulated antigen processing and presentation machinery, which was also associated with favorable patient survival in the TCGA ovarian cohort. T-cells specific against two mutated cancer-associated genes, NUP214 and JAK1, recognized autologous tumors. Gene-engineering with TCR from these neoantigen-specific T-cell clones conferred neoantigen-reactivity to peripheral T-cells.

Conclusions: Our study demonstrated the feasibility of efficiently identifying both $\mathrm{CD} 4^{+}$and $\mathrm{CD} 8^{+}$neoantigen-specific Tcells in EOC. Autologous lymphocytes genetically engineered with tumor antigen-specific TCR can be used to generate cells for use in the personalized adoptive T-cell transfer immunotherapy.

Keywords: Neoantigen, Ovarian cancer, CD4 ${ }^{+}$T-cells, CD8 ${ }^{+}$T-cells, Anti-tumor effect, T-cell receptor, Gene therapy

\footnotetext{
* Correspondence: song.liu@roswellpark.org;

junko.matsuzaki@roswellpark.org; kunle.odunsi@roswellpark.org

'Song Liu, Junko Matsuzaki, Lei Wei, Takemasa Tsuji and Sebastiano Battaglia contributed equally to this work.

'Department of Biostatistics and Bioinformatics, Roswell Park Comprehensive

Cancer Center, Buffalo, NY 14263, USA

${ }^{2}$ Center for Immunotherapy, Roswell Park Comprehensive Cancer Center,

Buffalo, NY 14263, USA

Full list of author information is available at the end of the article
}

(c) The Author(s). 2019 Open Access This article is distributed under the terms of the Creative Commons Attribution 4.0 International License (http://creativecommons.org/licenses/by/4.0/), which permits unrestricted use, distribution, and reproduction in any medium, provided you give appropriate credit to the original author(s) and the source, provide a link to the Creative Commons license, and indicate if changes were made. The Creative Commons Public Domain Dedication waiver (http://creativecommons.org/publicdomain/zero/1.0/) applies to the data made available in this article, unless otherwise stated. 


\section{Introduction}

Epithelial ovarian cancer (EOC) is the deadliest gynecological cancer, with approximately 22,240 new cases and 14,070 deaths in the United States for 2018. Approximately $80 \%$ of EOCs are diagnosed at an advanced stage, for which the standard treatment is surgery followed by platinum-taxane chemotherapy. Despite the initial efficacy of these standard of care approaches, the overall five-year survival probability is only $28 \%$ [1], and there is an enormous unmet need for the development of alternative therapies. Association between improved clinical outcome and increased intraepithelial $\mathrm{CD}^{+}$and/or $\mathrm{CD}^{+}$tumorinfiltrating lymphocytes (TILs) suggested antitumor roles of T-cells in EOC [2-5]. High-affinity neoantigen-reactive T-cells can escape negative selection in the thymus and might have a greater potential to evoke a multi-pronged anti-tumor immune response due to the lack of central tolerance against them [6]. Indeed, recent correlative clinical studies indicate that $\mathrm{T}$-cell reactivity to neoantigens is an important determinant of response to immune checkpoint inhibitors and other immunotherapies [7], suggesting that efforts to precisely define immunogenic neoantigens either for vaccination $[8,9]$ or adoptive T-cell therapy (ACT) $[10,11]$ could potentially provide clinical benefit [12].

A number of studies have reported T-cells specific to neoantigens in highly mutated tumors such as melanoma and lung cancer [8, 13-19]. Results from the existing studies of spontaneous $\mathrm{T}$-cell response to tumor neoepitopes in EOC have been mixed [20-23]. Two earlier studies suggested that EOCs are rarely/unlikely to elicit neoepitope specific spontaneous $\mathrm{T}$-cell response due to the relatively low somatic mutation burden [20, 21]. However, these studies were either limited by the small sample size (1-3 patients) or carried out in a murine ovarian tumor model, which has the concern of neoantigen being silenced and/or lost due to immune editing in the immunocompetent mouse. The studies were also restricted to $\mathrm{CD}^{+}$T-cells only, leaving the landscape of spontaneous $\mathrm{CD}^{+}{ }^{+} \mathrm{T}$-cell responses to tumor neoepitopes unexplored. Two more recent studies with larger sample size implied that by comprehensively screening all possible neoantigen candidates, identification of neoepitope specific T-cells is achievable in EOCs $[22,23]$. Of the 1714 and 776 putative mutated neoantigens screened, less than 0.5 and $2 \%$ were shown to be immunogenic in validation experiments, respectively. Moreover, the capacity of autologous ovarian tumor recognition by neoantigen-specific $\mathrm{T}$-cells has not been addressed.

The aim of this study was to investigate whether the validation rate of neoantigen identification in EOCs can be significantly improved through in silico prioritization. Whole-exome and transcriptome sequencing analysis of treatment-naive EOC patients were performed to identify neoantigen candidates, and the immunogenicity of prioritized neoantigens was evaluated by analyzing spontaneous neoantigen-specfic $\mathrm{CD}^{+}$and $\mathrm{CD}^{+}$T-cell responses in the tumor and/or peripheral blood. The biological relevance of neoantigen-specific T-cell lines and clones were analyzed by evaluating the capacity of autologous ovarian tumor recognition. Genetic transfer of T-cell receptor (TCR) from these neoantigen-specific T-cell clones into peripheral blood T-cells was conducted to generate neoepitope-specific T-cells. The molecular signature associated with positive neoantigen T-cell responses was investigated, and the impacts of expression level and lymphocyte source on neoantigen identification were discussed .

\section{Results}

\section{Patient characteristics}

The characteristics of the study cohort are shown in Additional file 12: Table S1. All 20 treatment-naïve patients underwent maximal debulking surgery (85\% were optimally debulked, with $45 \%$ complete resection) from which the tumor tissue were procured. Patients in this cohort had typical characteristics of advanced EOC cases: median age at diagnosis of 60 (range 44 to 89), high stage (IIIC, IV; 100\%), and the majority with high grade serous histology (75\%). The median duration of follow-up was 29.7 months. The median progression-free survival was 18.1 months and the median overall survival was 30.9 months.

\section{Mutational landscape}

Whole-exome sequencing (WES) was performed on 22 pre-therapy biopsies and matched normal samples (Peripheral blood mononuclear cells, PBMC) from the 20 EOC patients, as the first step in our workflow for neoantigen discovery and prioritization (Fig. 1a). The specimens consisted of primary tumor in 9 patients, locally invasive tumor in 9 patients, paired primary and locally invasive tumors in 2 patients. Somatic mutations were identified by comparing the tumor with the matched blood DNA as described [24, 25]. We identified a total of 2096 somatic mutations from the 20 patients, including 1368 non-synonymous somatic mutations (median $=62$ ), and the number of genes with altered amino acid sequence ranged from 9 to 183 per patient (Fig. 1b). TP53 was mutated in 16 patients, including 7 truncating mutations predicted to cause loss-of-function (Additional file 1: Figure S1). Nine genes were mutated in 3 out of 20 patients, including two known Cancer Gene Census (CGC) genes [26]: NF1 and STAG2. Of these nine genes, $I L 27 R A$ appears to be interesting as two of the three mutations were truncating mutations. The two IL27RA loss-of-function mutations were both identified from locally invasive tumor while the third IL27RA missense mutation 

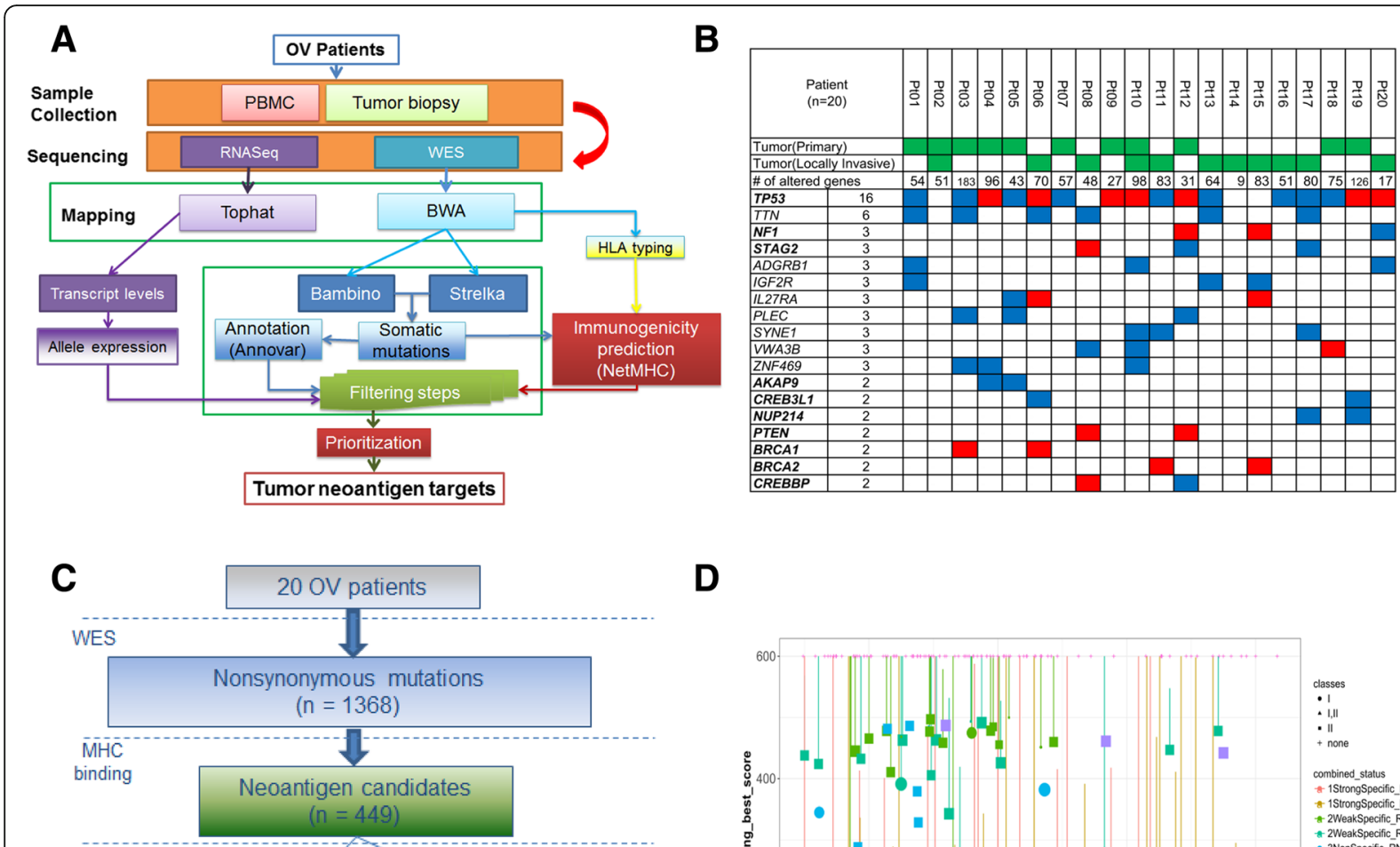

\section{D}

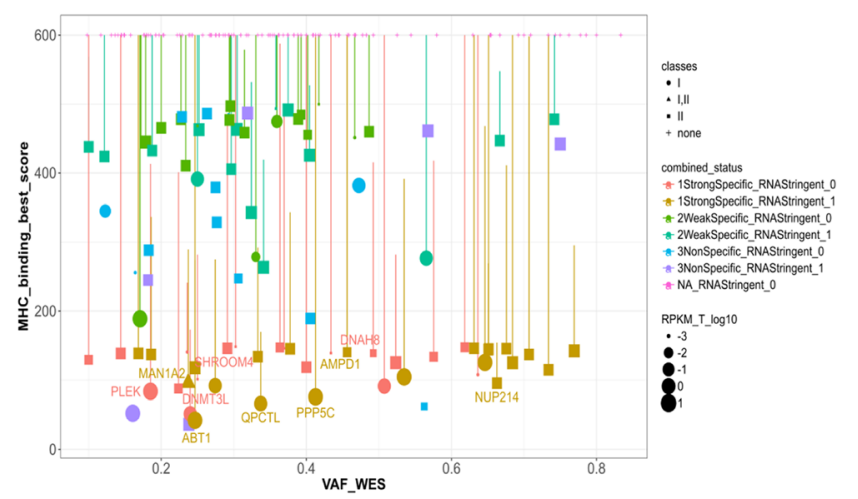

Fig. 1 Integrative genomics and bioinformatics approach for neoantigen discovery and prioritization. a Overview of next-generation sequencing and neoantigen prediction. Whole-exome sequencing was performed on the pretreatment tumor and matched normal samples to identify somatic mutations, which were applied in neoantigen prediction pipeline that evaluates MHC binding, clonal status and gene expression to generate neoantigen specific to the patient's HLA haploytype (Methods). b Top recurrently mutated genes in the $20 \mathrm{EOC}$ patients, ordered by the numbers of recurrence. Known Cancer Gene Census (CGC) genes are in bold. For genes with recurrence at least 3, all genes are included. For genes with recurrence equals to 2 , only known CGC genes are included. Red: truncating mutations, including nonsense SNV or frameshift Indels; Blue: altering mutations, including missense SNV or in-frame Indels. c Summary of neoantigen predictions in the 20 EOC patients, stratified by the MHC class type and gene expression status. There are 100 neoantigen predicted to bind to MHC class I only, 234 to class II only, and 115 to both class I and II, respectively. Among them, 209 are expressed based on RNAseq data. $\mathbf{d}$ The neoantigen landscape of Pt \#19, as displayed in the Christmas Light Plot (CLP). The CLP incorporate pre-defined criteria for neoantigen prioritization, including MHC binding affinities, expression level, HLA class types, and the mutant clonal status. X-axis: Variant allele fraction (VAF) in WES, which can be used to infer clonal status; Y-axis: the predicted binding affinity of the mutant peptide. Each dot represents a neoantigen with the following characteristics displayed; size: the gene expression level by RNAseq; shape: HLA binding classes (I, II, or both); vertical bar: difference between mutant and wildtype binding affinities; color: stratified based on the mutant versus wildtype binding and mutant expression level (Methods). Gene symbols are displayed for neoantigens selected for screening

was found in primary tumor. In addition, there are 70 genes mutated in two patients, including seven CGC genes (Additional file 13: Table S2). PTEN, BRCA1, and $B R C A 2$ were each mutated in 2 patients, with all of them loss-of-function mutations. There was no gene found to be mutated at a significantly different frequency between primary and locally invasive tumors. In the two patients with both primary and locally invasive tumors, we compared every mutation's variant allele fraction (VAF) between the two tumors and showed they were overall highly consistent (Additional file 2: Figure S2a).

\section{Identification of neoantigens}

Candidate neoantigens were identified using the computational pipeline as outlined in Fig. 1a. We identified neoantigen candidates as mutations harboring mutant peptide whose binding affinity to patients' MHC was not only strong $(<150 \mathrm{nM})$, but also specific (i.e., higher affinity than that of matched wild-type peptide). The second condition was included to enrich highly immunogenic neoepitopes because some neoepitopereactive TCRs were considered to cross-react to the corresponding wild-type epitope if both mutated and wild-type epitopes were similarly presented [27]. In this 
case, T-cell precursors expressing such TCRs are expected to be eliminated in the thymus, which would result in lower precursor frequencies in the periphery. A total of 449 neoantigen candidates were found to have at least one predicted neopeptide with strong and specific MHC I and/or II-binding affinity. The number of predicted neoantigen in each patient ranged from 4 to 75 , with a median of 21 (Additional file 14: Table S3). In the two patients with primary/locally-invasive tumor pair, the majority of neoantigens were shared by both primary and locally invasive tumors (100 and $77.8 \%$, respectively) (Additional file 2: Figure S2b).

These 449 neoantigens included 215 which contained MHC I-binding neopeptides, and 349 which contained class II-binding neopeptides (Fig. 1c). One quarter (115 of 449) of neoantigens were predicted to harbor neopeptides which bind to both class I and II. We classified these 449 neoantigens based on the mutant allele's expression level in RNAseq data (see Methods). About half (209) neoantigens demonstrated robust expression of the mutant allele while the rest (240) did not.

\section{Prioritization of neoantigens}

To prioritize neoantigen candidates for peptide synthesis and T-cell assays, we ranked all predicted neoantigens within each patient based on a predefined set of criteria: 1) mutations in CGC genes; 2) MHC binding affinity of the mutant peptide; 3 ) difference in the binding affinities between mutant and the matched wild type peptides; 4) variant allele fraction (VAF) of the mutation; 5) expression level including both the mutant allele and the overall level of the gene; 6) type of MHC binding (class I only, class II only, or both class I and II). To facilitate this process, we designed a specific type of visualization plot (Christmas Light Plot, or CLP) incorporating all these types of information (Fig. 1d). The final selection of neopeptides involved a target selection board that evaluated the target peptides based on the criteria described above, with additional considerations of biochemical properties related to peptide synthesizability. For the ten patients with autologous PBMC, tumorderived single-cell suspension and tumor biospecimens available, a total of 75 neopeptides were selected for synthesis, with a median of 7 and a range of 3-12 neopeptides per patient (Additional file 15: Table S4). These include 36, 32, and 7 neopeptides that are predicted to bind to class I only, class II only, and both class I and II, respectively. Twenty five of these 75 neopeptides did not demonstrate robust expression of the mutant allele in RNAseq (Methods), and they were included to investigate the relationship between expression level and induction of $\mathrm{T}$-cell response.

\section{Evaluation of neoepitope immunogenicity}

The immunogenicity of the neoepitopes was evaluated in the ten patients from whom live T-cells from both PBMC and tumors were available. $\mathrm{CD}^{+}$and $\mathrm{CD}^{+} \mathrm{T}$ cells were isolated from each specimen and stimulated with T-cell-depleted PBMCs as antigen-presenting cells (APCs) that had been pulsed with pooled neoepitopes. T-cell-depleted PBMCs were used to enrich APCs such as dentritic cells, monocytes/macrophages and B cells. A total of 27 IFN- $\gamma$-producing T-cell responses were detected in samples from 5 of 10 patients, including 20 responses against 10 individual neopeptide and 7 responses against 4 pooled neopeptides (Fig. 2a). These positive T-cell responses were highly mutant-specific, with the reactivity against mutated peptide at least twofold greater than the corresponding wild-type peptide (Additional file 3: Figure S3 and Additional file 4: Figure S4). Both $\mathrm{CD}^{+}$and $\mathrm{CD}^{+}{ }^{+} \mathrm{T}$-cells showed neoepitopespecific responses, with 13 responses mediated by $\mathrm{CD} 8^{+}$ T-cells and 14 by $\mathrm{CD} 4^{+} \mathrm{T}$-cells. The median number of positive $\mathrm{T}$-cell responses against individual neopeptides was 4 in the 5 responders, with a median of 2 reactive neoepitopes per patient.

For the 9 neopeptides (or neopeptide pools) that were recognized by $\mathrm{CD}^{+}$T-cells, 5 exclusively elicited mutant-specific T-cell response in either TILs or PBMCs. Likewise, 4 of the 9 neopeptides (or neopeptide pools) that were recognized by $\mathrm{CD} 4^{+} \mathrm{T}$-cells exclusively elicited mutant-specific T-cell response in either TILs or PBMCs. This is consistent with prior evidence that there exists a discordance of neoepitope recognition between TILs and PBMCs [22]. Examples included the CD4 $4^{+} \mathrm{T}-$ cell response against mutant epitopes of JAK1 which were detected only in TILs, and the CD8 ${ }^{+}$T-cell response against mutant epitopes of TRPC4 which was present only in PBMCs (Fig. 2a). On the other hand, 18 of the 27 responses were detected in both TILs and PBMCs, indicating that both types of patient's specimen are useful for identifying neoantigen-reactive T-cells (Fig. 2b). Examples include $\mathrm{CD}^{+}$and $\mathrm{CD}^{+}{ }^{+} \mathrm{T}$-cell responses against IL27RA2 which were detetcted in both PBMC and TILs. Among the four patients with detected T-cell responses in both TILs and PBMCs, the responses were detected higher in TILs than PBMCs in three patients; while in $\mathrm{Pt} \# 5$, there were more response in PBMCs than TILs (Fig. 2b). In order to explore immunosuppression status of TILs, we analyzed the expression level of a panel of 10 immune inhibitory molecules from the tumor RNA-Seq data of these four patients. These immune inhibitory molecules include PD1, PDL1, CTLA4, CD80, CD86, LAG3, TIM3, LAGLS9, MYC and FOXP3. Remarkably, Pt \#5 showed higher expression of all these immune inhibitory genes than those of the other three patients (Additional file 5: Figure S5). 


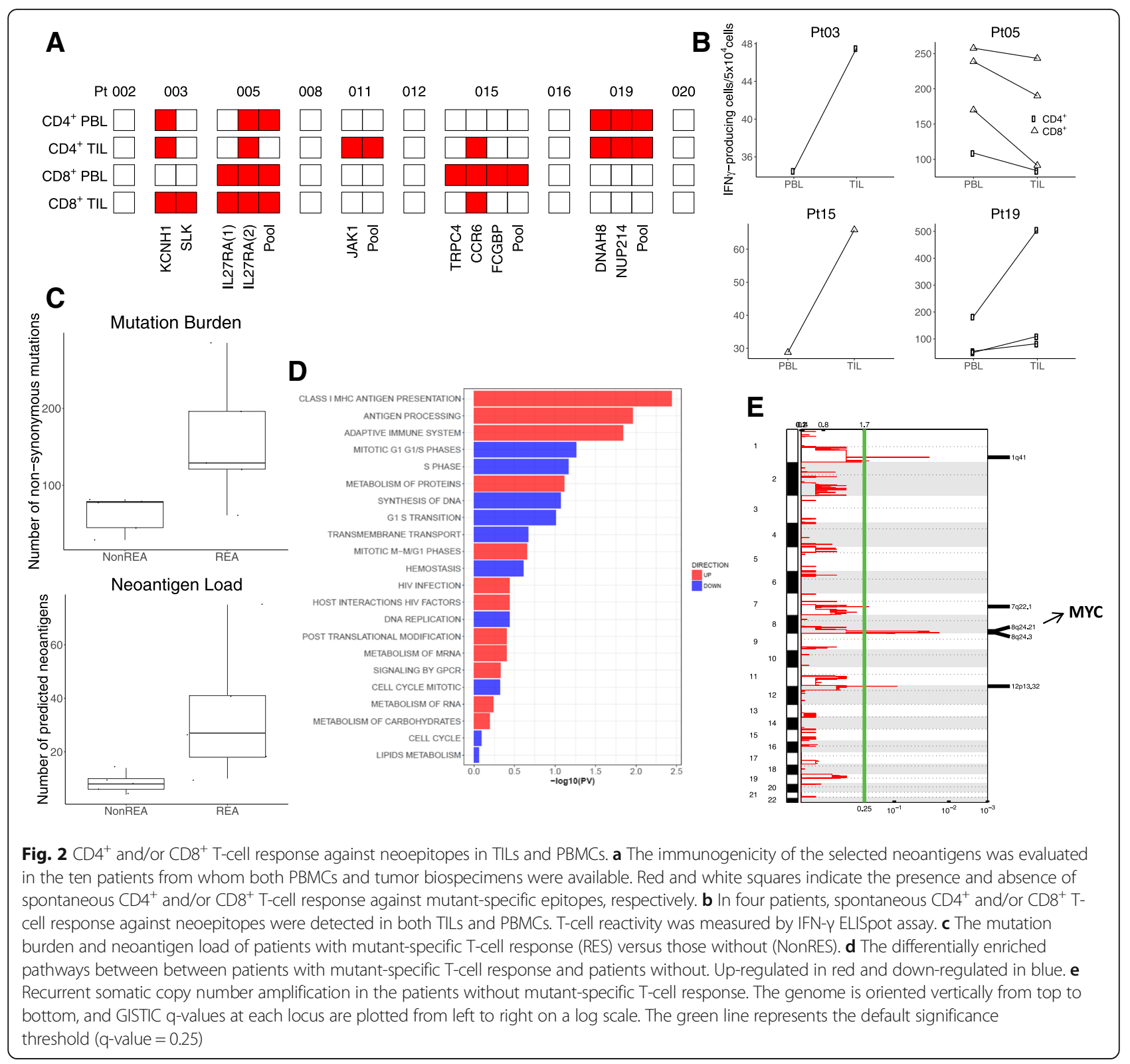

In total, 7/50 (14\%) predicted neoepitopes that showed robust expression in RNAseq data induced T-cell responses. Interestingly, T-cell responses were also detected against $3 / 25(12 \%)$ predicted neopeptides, $\mathrm{KCNH} 1$ in Pt \#3, TRPC4 in Pt \#15 and DNAH8 in Pt \#19, that were not robustly expressed in RNAseq (Additional file 15: Table S4). These 3 genes were all weakly expressed (with RPKM less than 1), although it is possible that the sequence depth of our RNAseq might not be sufficient to detect the mutant allele. It has been suggested that high level of expression may not be required for inclusion of a neoantigen candidate [28], based on the observations that very low levels (e.g., even a single peptide-HLA complex) may be sufficient for a cell to elicit a cytolytic T-cell response [29].
Signatures of neoepitope-specific T-cell response

As expected, we found that patients with neoantigenspecific T-cell responses have significantly higher mutation burden and neoantigen load than those without (Fig. 2c). The median number of nonsynomous mutation is 84 in patients with responses, compared with 49 in patients without responses $(p=0.026$, one-tailed t-test). The corresponding number of predicted neoantigen is 27 and 8 , respectively ( $p=0.043$, one-tailed t-test). Interestingly, patients with positive T-cell responses are significantly enriched for $B R C A 1 / 2$ somatic mutations (3/5 vs $0 / 5, p=0.038$, chi-square test). When gene expression profiles for responders and non-responders were compared, the most significantly and differentially enriched pathways in responders were related to antigen 
processing and presentation machinery (APPM), suggesting that not only the number of neoantigens, but also antigen processing and presentation in tumor regulate generation of $\mathrm{T}$-cell responses against neoantigens (Fig. 2d). On the other hand, the non-responders were characterized with a unique signature of $M Y C$ amplification (Fig. 2e, Additional file 6: Figure S6), which was recently shown to promote immune evasion through the modulation of immune regulatory molecules [30].

By intersecting the list of differentially expressed genes (responders versus non-responders) with the genes of the APPM pathways, an 31-gene signature of APPM was derived (Fig. 3a, Additional file 16: Table S5). Based on median expression value of the APPM signature, we stratified TCGA ovarian cancer patients into groups of high vs low expression of this signature (Fig. 3b). In consistent with prior evidence that antigen presentation pathway is reduced in high-risk ovarian cancer [31], patients with higher expression of APPM signature have longer overall survival than patients with lower expression of the signature $\left(p=5 \times 10^{-4}\right.$, Cox ProportionalHazards Model) (Fig. 3c). Furthermore, patients with higher expression of APPM signature exhibited higher levels of $\mathrm{CD} 4^{+}$memory T-cells, Th1 and Th2 $(p=1.2 \times$ $10^{-3}, 1 \times 10^{-13}$, and $3.1 \times 10^{-3}$, respectively, t-test), and lower level of Tregs $\left(p=4 \times 10^{-6}\right.$, t-test) in tumors (Fig. $3 \mathrm{~d})$. On the other hand, patients with lower expression of APPM signature have a modest but significant increase in $M Y C$ expression $\left(p=1.7 \times 10^{-4}\right.$, t-test) (Fig. 3e) . Consistent with these findings, the TCGA ovarian cancer patients displayed a negative association between the expression levels of APPM signature and MYC ( $p=$ $1.37 \times 10^{-6}$, linear regression) (Fig. 3f). Similar trends were observed in the EOC patients from our study cohort, where the small sample size limited their statistical significance.

\section{Characterization of neoepitope-specific T-cells}

In order to further characterize neoepitope-specific $\mathrm{T}$ cells in EOC, we established neoantigen-specific T-cell lines by isolating and expanding peptide-reactive T-cells. $T$-cell lines that specifically recognized mutated peptides were established in 3 out of 9 cases we attempted. Based on the availability of autologous tumor specimens, we focused on NUP214 neoepitope-specific $\mathrm{CD}^{+}{ }^{+} \mathrm{T}$-cells obtained from TILs of Pt \#19 and JAK1 neoepitopespecific CD4 ${ }^{+}$T-cells obtained from TILs of Pt \#11.

About $80 \%$ of neoepitope-specific $\mathrm{CD}^{+}{ }^{+} \mathrm{T}$-cell line produced IFN- $\gamma$ against the mutated NUP214 peptide but not the corresponding wild-type peptide (Fig. 4a).

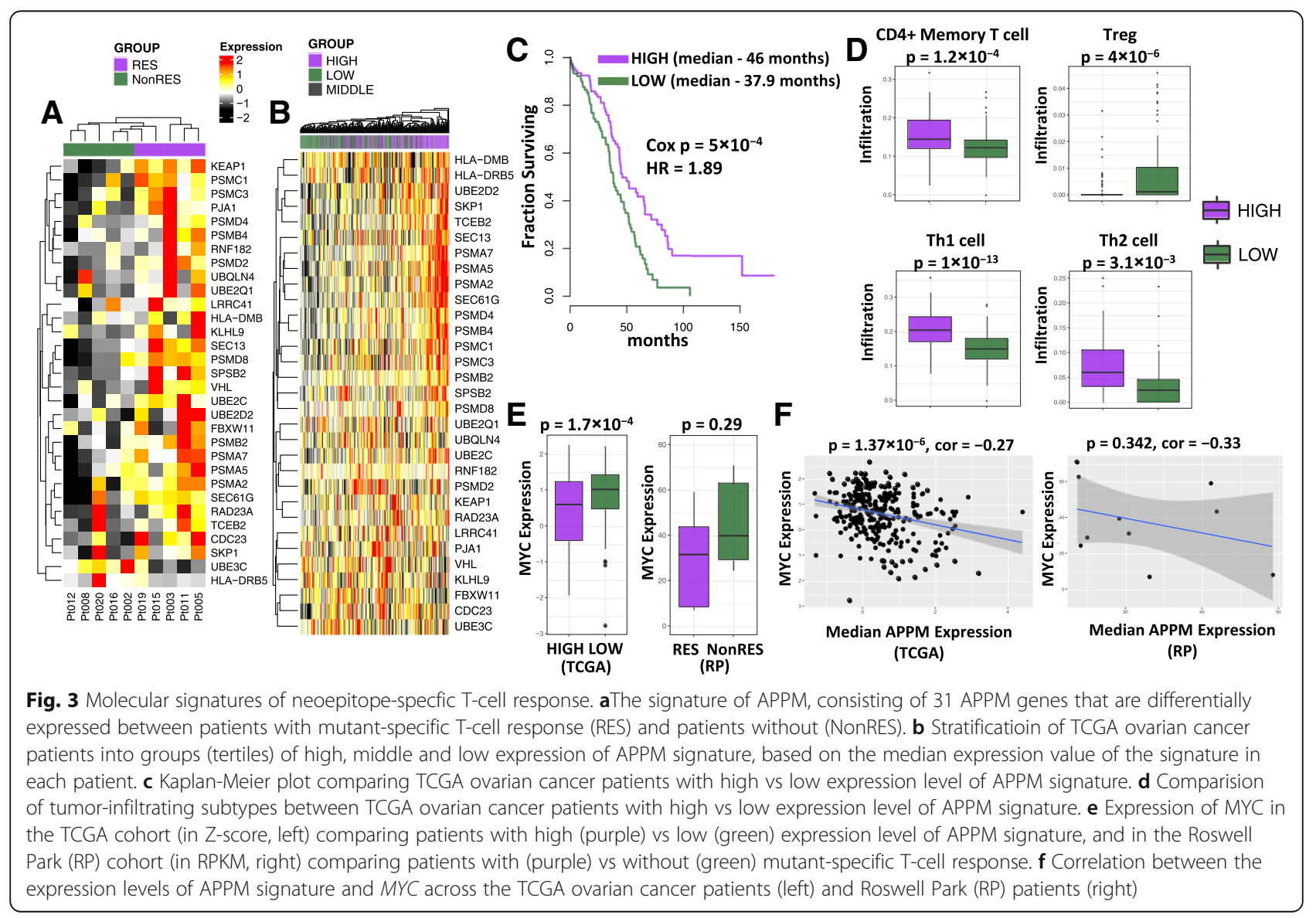




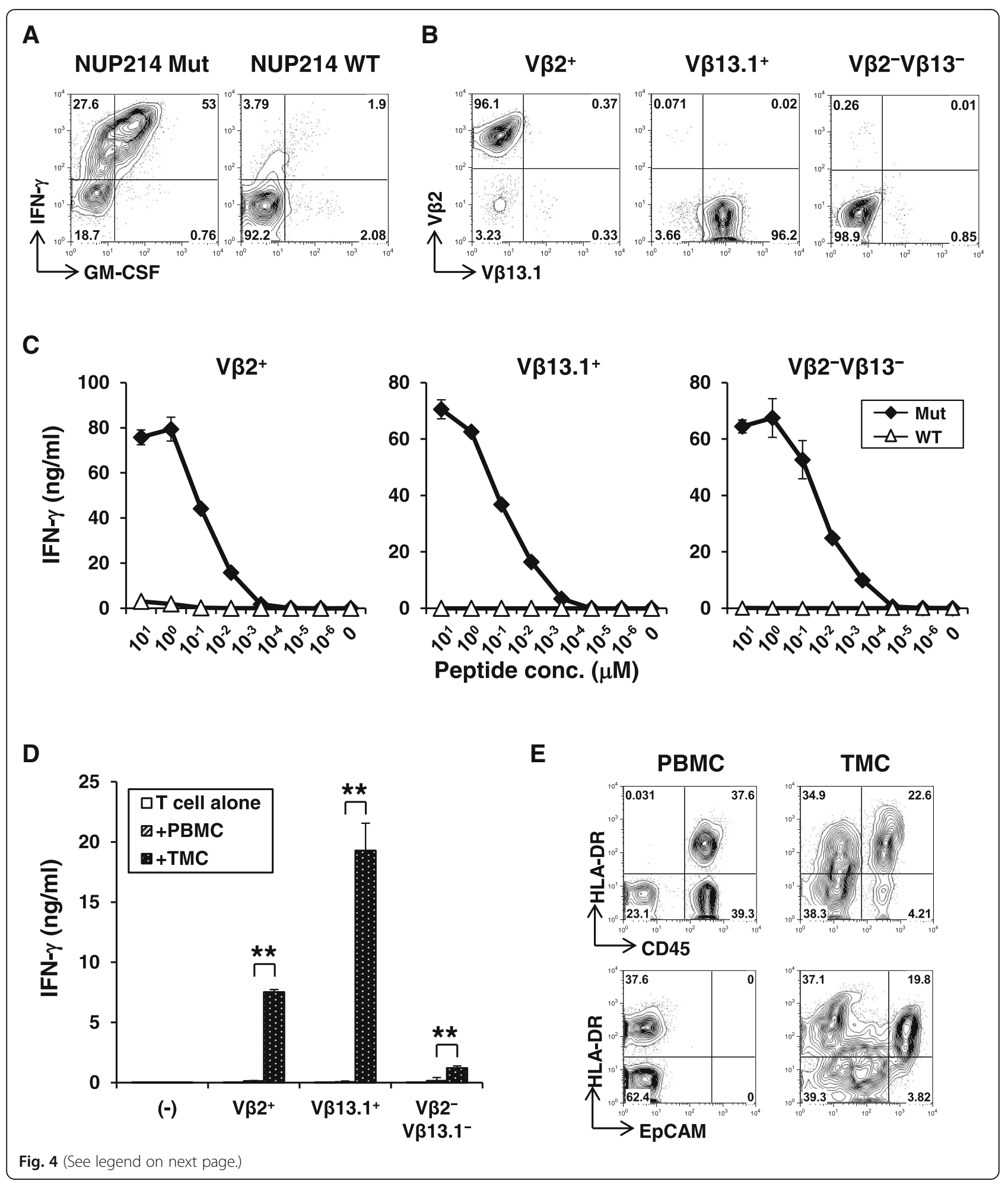


(See figure on previous page.)

Fig. 4 Characterization of NUP214 neoepitope-specific CD4 ${ }^{+}$T-cells. a Peptide reactivity of a NUP214 neoepitope-specific CD4 ${ }^{+}$T-cell line. IFN- $\gamma$ and GM-CSF production on CD4 ${ }^{+}$T-cells against mutated or wild-type NUP214 peptide-pulsed autologous EBV-transformed B (EBV-B) cells were determined by intracellular cytokine staining. $\mathbf{b}$ Establishment of NUP214 neoepitope-specific CD4 ${ }^{+}$T-cell clones. TCR V $\beta 2^{+}, V \beta 13.1^{+}$, or V $\beta 2^{-} V B 13.1^{-}$cells in the NUP214 neoepitope-specific CD4 ${ }^{+}$T-cell lines were isolated. After expansion, each T-cell clone was stained by TCR V $\beta$ subtype-specific antibodies. c Avidity of NUP214 neoepitope-specific T-cell clones. V $\beta 2^{+}, \mathrm{V} \beta 13.1^{+}$, and V $\beta 2^{-} \mathrm{V} \beta 13.1^{-} \mathrm{CD} 4^{+} \mathrm{T}_{\text {-cell clones }(50,000}$ cells) were stimulated with autologous EBV-B cells (25,000 cells) pulsed with NUP214 mutated or wild-type peptide in a 96-well round bottom plate for $24 \mathrm{~h}$. IFN- $\gamma$ level in the culture supernatant was measured by ELISA. The data represents mean \pm s.d. of duplicate wells. $\mathbf{d}$ Reactivity of $\mathrm{V} \beta 2^{+}, \mathrm{V} \beta 13.1^{+}$, and $\mathrm{V} \beta 2^{-} \mathrm{V} \beta 13.1^{-}$T-cell clones against autologous tumor cells. PBMCs or TMCs $\left(100,000\right.$ cells) were co-cultured with $\mathrm{V} \beta 2^{+}, \mathrm{V} \beta 13.1^{+}$, or VB2-VB13.1- NUP214 neoepitope-specific CD4 ${ }^{+}$T-cells (50,000 cells) or without T-cells (-) for $24 \mathrm{~h}$. TMCs: tumor tissue-derived mononuclear cells. IFN- $\gamma$ production was measured by ELISA. The data represent mean + s.d. of triplicate wells. ${ }^{* *} p<0.01$ (student's t-test) compared to IFN- $\gamma$ level against PBMCs. e Expression of MHC class II on CD45 immune cells and EpCAM+ tumor cells. HLA-DR expression on CD45 ${ }^{+}$or EpCAM ${ }^{+}$ cells from PBMCs or TMCs were analyzed by flow cytometry

Low-resolution TCR V $\beta$ spectratyping identified the $\mathrm{CD}^{+}{ }^{+} \mathrm{T}$-cell response as oligoclonal (Additional file 7 : Fig. S7a), composed of $20 \% \mathrm{~V} \beta 2^{+}$and $45 \% \mathrm{~V} \beta 13.1^{+} \mathrm{T}-$ cells, respectively. Approximately $30 \%$ of cells were of other $\mathrm{V} \beta$ subtype not identified by this antibody panel. Combination of V $\beta$-staining and intracellular IFN- $\gamma$ staining demonstrated that all 3 distinct major subsets recognized the same neopeptide (Additional file 7: Fig. S7b). Therefore, TCR V $\beta 2^{+}, \mathrm{V} \beta 13.1^{+}$, or $\mathrm{V} \beta 2^{-} \mathrm{V} \beta 13.1^{-}$ cells were further isolated by flow cytometric cell sorting to obtain clonal populations (Fig. 4b). Avidity for recognition of mutated peptide by $\mathrm{V} \beta 2^{+}, \mathrm{V} \beta 13.1^{+}$, and $\mathrm{V} \beta 2^{-} \mathrm{V} \beta 13.1^{-}$NUP214-specific $\mathrm{CD}^{+}{ }^{+}$T-cell clones was similar (Fig. 4c). Responses were strictly specific for mutation as there was no recognition of wild-type peptide even at higher concentrations. As NUP214-specific $\mathrm{CD}^{+}{ }^{+} \mathrm{T}$-cell responses was detected in the tumor from Pt \#19 (Fig. 2 a), we reasoned that the mutated NUP214 epitope was naturally presented in the tumor microenvironment. Therefore, we tested whether NUP214specific $\mathrm{CD}_{4}{ }^{+} \mathrm{T}$-cells are activated by autologous tumor cells. Indeed, NUP214 neoepitope-specific CD4 ${ }^{+}$T-cells produced IFN- $\gamma$ specifically against tumor cells, but not against autologous PBMCs (Fig. 4d). These results strongly support that NUP214-specific CD4 ${ }^{+}$T-cells were activated in the tumor microenvironment. In Pt \#19 tumor, both $\mathrm{CD}_{4} 5^{+}$hematopoietic cells and $\mathrm{EpCAM}^{+}$ EOC expressed MHC class II (HLA-DR) (Fig. 4e). Therefore, both direct presentation by cancer cells and indirect cross-presentation of tumor-derived NUP 214 by hematopoietic antigen-presenting cells are possible as a mechanism for activation of mutated NUP214-specific $\mathrm{CD}^{+}{ }^{+} \mathrm{T}$-cells in the tumor microenvironment.

The JAK1 neoepitope-specific $\mathrm{CD}^{+}$T-cells were isolated and expanded (Additional file 8: Figure S8a) and were analyzed for TCR V $\beta$ usage (Additional file 8: Figure S8b). The majority (75\%) of JAK1-specific CD $4^{+}$ T-cell line was $\mathrm{V} \beta 13.6^{+}$, indicating a monoclonal response. TCR V $\beta 13.6^{+} \mathrm{CD}^{+} \mathrm{T}$-cells were further isolated and expanded for functional analyses (Additional file 8: Fig. S8c). V $\beta 13.6^{+} \mathrm{CD}^{+} \mathrm{T}$-cells specifically recognized
JAK1 mutant peptide over the corresponding wild-type peptide (Additional file 8: Figure S8d). Because autologous tumor mononuclear cells (TMCs) were not available for this patient, we tested the reactivity against tumor ascites mononuclear cells (AMCs) and found that $\mathrm{CD}^{+}{ }^{+} \mathrm{T}$-cells produced IFN- $\gamma$ when co-cultured with the AMCs but not with the autologous PBMCs (Additional file 8: Figure S8e).

\section{Generation of neoepitope-specific T-cells by TCR gene- engineering}

To test whether neoantigen-reactivity is solely mediated by TCR and whether neoantigen-specificity can be transferred to other T-cells by TCR gene-engineering, we first cloned TCR gene from 5 neoepitope-specific T-cell clones (3 mutated NUP214-specific CD4-TCR from Pt \#19, 1 JAK1-specific CD4-TCR from Pt \#11, and 1 TRPC4-specific CD8-TCR from Pt \#15) into a retroviral plasmid vector (Fig. 5a) [32]. To test the functionality of the cloned TCR, peripheral T-cells from a healthy donor were polyclonally activated and transduced by the TCRexpressing retroviral vectors. In $4 / 5$ cases, TCR geneengineered T-cells were demonstrated for mutated peptide-specific reactivity. In the case of 3 NUP214specific TCRs, two TCRs from $\mathrm{V} \beta 13.1^{+}$or $\mathrm{V} \beta 2^{-} \mathrm{V} \beta 13.1^{-}$ T-cell clones provided neoepitope-specific reactivity (Fig. $5 \mathrm{~b}-\mathrm{c}$ ), while that from $\mathrm{V} \beta 2^{+} \mathrm{T}$-cell clone did not despite of similar reactivity by the parental T-cell clones (Fig. 4c). Both $\mathrm{CD}^{+}$and $\mathrm{CD}^{+}$T-cells transduced with NUP214-specific TCR showed reactivity against the neoepitope (Additional file 9: Figure S9). Similar observations were made for mutated JAK1-specific TCR, where we have established high-titer retroviruspackaging PG13 cell clone. After 2 transductions, nearly 60\% T-cells expressed transduced TCR, as determined by the increase in TCR VB13.6 ${ }^{+}$expression (Fig. 5d). Mutated JAK1-specific TCR-transduced T-cells also displayed strong and specific reactivity against the mutated JAK1 peptide (Fig. 5e-f). In addition to $\mathrm{CD}^{+}{ }^{+} \mathrm{T}$-cells, we also cloned TCR gene from TRPC4 neoepitope-specific $\mathrm{CD}^{+} \mathrm{T}$-cells, and confirmed the neoantigen reactivity by 


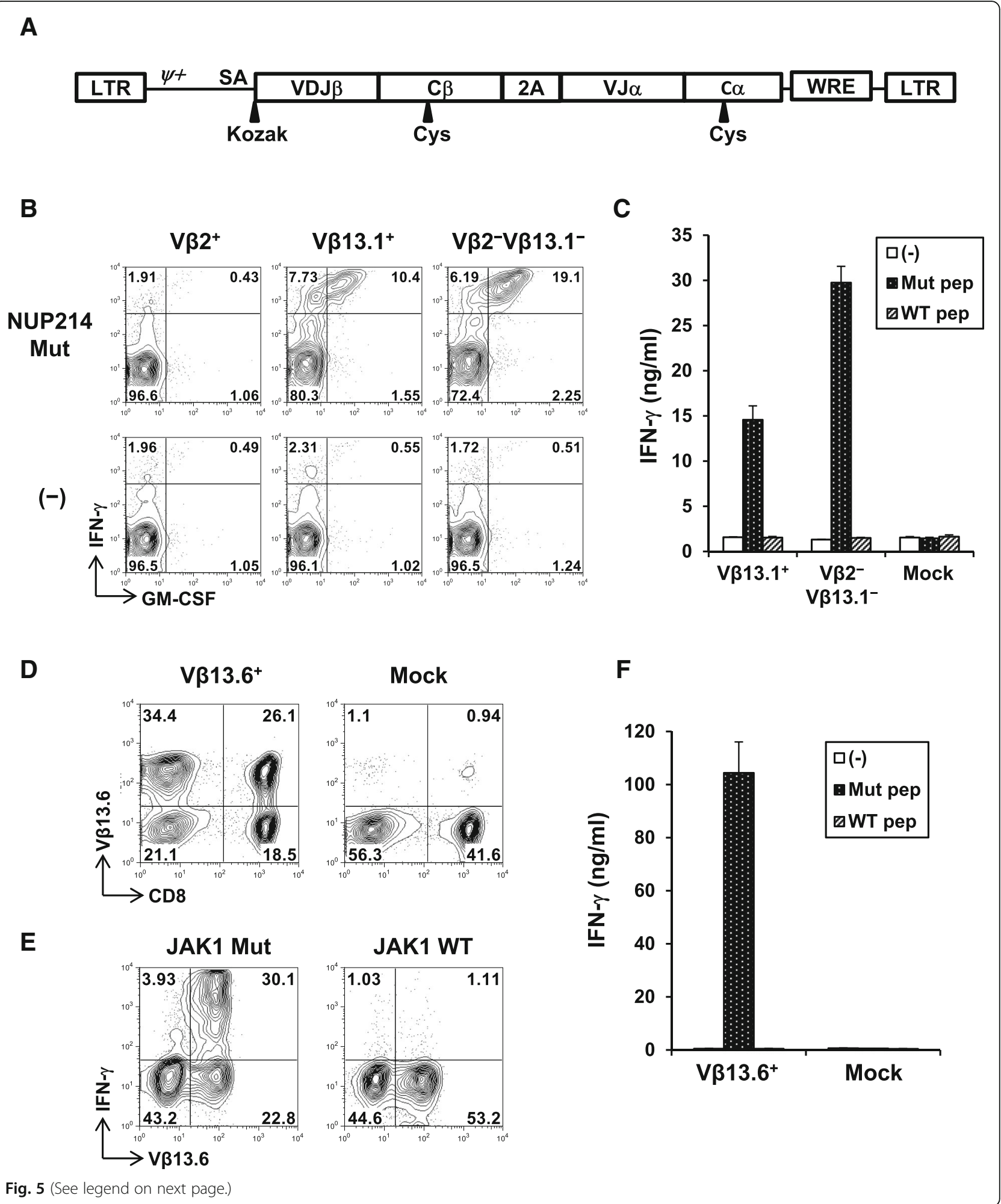

Fig. 5 (See legend on next page.) 
(See figure on previous page.)

Fig. 5 Generation of neoantigen-specific CD4 ${ }^{+}$T-cells by TCR gene-engineering. a Schematic representation of retroviral TCR expression vector for TCR gene-engineering. LTR: long terminal repeats; $\psi^{+}$: extended packaging signal; SA: Splice acceptor site from the first exon-intron junction of human elongation factor-1a; Kozak: Kozak consensus sequence (GCCACC); VDJ $\beta$ : TCR $\beta$ chain variable-diverse-joining regions; C $\beta$ : TCR $\beta$ chain constant region containing a Cystein modification; 2A: the P2A translational skipping sequence; VJa: TCR a chain variable-joining regions; Ca: TCR a chain constant region containing a Cystein modification; and WRE indicates the: Woodchuck hepatitis virus posttranscriptional regulatory element. b-c T-cell function of NUP214-specific TCR-transduced T-cells. b IFN- $\gamma$ and GM-CSF production from V $\beta 2^{+}$, V $\beta 13.1^{+}$, or V $\beta 2^{-}$V $\beta 13.1^{-}$TCRtransduced T-cells against autologous EBV-B cells pulsed with or without NUP214 mutated peptide. $\mathbf{c}$ IFN- $\gamma$ production from V $\beta 13.1^{+}$or $V \beta 22^{-} V \beta 13.1^{-}$ TCR transduced T-cells against NUP214 mutated or wild-type peptide was measured by ELISA. Mock: TCR-untransduced T-cells. d-f Transduction efficiency and function of JAK1 neoepitope-specific TCR-transduced T-cells. d V $\beta 13.6^{+}$TCR transduction efficiency was examined by flow cytometry. e Detection of JAK1 neoepitope-specific response on V $313.6^{+}$T-cells by intracellular cytokine staining. $\mathbf{f}$ Reactivity of TCR-transduced T-cells against JAK1 mutated or wild-type peptide was tested by ELISA

TCR gene-engineered T-cells (Additional file 10: Figure S10).

\section{Discussion}

Advanced EOC is a highly lethal disease, with a dismal five-year survival rate below $30 \%$ [1]. The strong association of TILs with better survival [33], which is counter-regulated by innate and adaptive immune escape mechanisms such as expression of immunosuppressive molecules and recruitment of immunosuppressive cells, indicates that patients with advanced EOC might benefit from immunotherapy. Emerging immunotherapies targeting patient-specific neoantigens have attracted considerable interest because immune responses to tumor-specific neoantigens are less constrained by central and peripheral immune tolerance mechanisms [34].

Given the relatively lower mutation burden of EOC than melanoma and lung cancer, previous investigations of spontaneous T-cell response to tumor neoepitope in EOC have taken the approach of comprehensively screening all identified neoantigen candidates [20-23]. For example, Bobisse et al screened all 776 predicted HLA class I-binding neopeptide individuals as well as 19 neopeptide pools from 19 patients (mean 46 per patient) and validated 15 of them, or 2\% [22]. Dinger et al screened all 1714 putative mutated neoantigens from 7 patients (mean 214 per patient) and validated 8 of them, or $0.5 \%$ [23]. In contrast, we only screened a subset (mean 9 per patient) of neopeptide candidates prioritized by our in silico approach, and achieved a substantially higher validation rate (14 of the 75 neopeptide individuals and 10 neopeptide pools, or 19\%) for practical use, especially when limited patient-derived specimens are available. On the other hand, our approach might have missed some bona fide neoantigen targets, and therefore underestimated the prevalence of neoepitope specific Tcells in patients with EOC.

As expected, patients exhibiting $\mathrm{T}$-cell responses against neoepitopes were found to have significantly higher mutation burden and neoantigen load. In addition, these responders tended to have higher BRCA1/2 mutation rate than those without. Due to the relatively low mutation burden of EOC and widespread interpatient tumour heterogeneity, our prioritization approach will more likely be applicable to a subset of patients of EOC. In addition, since somatic mutations can vary at different times in tumor evolution (e.g., primary tumor versus tumor relapse), it will be desirable to repeat this procudure sequentially to identify new neoepitope-specific $\mathrm{T}$ cells.

We found elevated level of APPM signature in patients who spontaneously developed neoepitope-specific T-cell response, while MYC amplification was observed in patients who did not. Among the five responders in our study, Pt \#5 has lower mutation burden than three of the five non-responders (45 vs $49-52$, Fig. 2c). Interestingly, the expression level of APPM signature is almost two-fold higher in Pt \#5 than the three non-responders (Additional file 11: Figure S11). Our results provided independent edvidence that antigen presentation pathway is associated with ovarian cancer prognosis [31], and lends additional support for the crucial role of this machinery in TIL infiltration and defining $\mathrm{CD}^{+} / \mathrm{CD}^{+} \mathrm{T}$ cell responses against neoantigens.

The biological relevance of patient-derived T-cells against neoantigens from known cancer-associated genes, NUP214 and JAK1, were confirmed by the demonstration of autologous tumor recognitions. NUP214 encodes a protein localized to the cytoplasmic face of the nuclear pore complex where it is required for proper cell cycle progression and nucleocytoplasmic transport. NUP214 forms a fusion gene with the DEK gene on chromosome 6 in a $t[6,9]$ translocation associated with acute myeloid leukemia and myelodysplastic syndrome $[35,36]$. Somatic mutations in $J A K 1 / 2$ have been proposed as a mechanism of evasion to immune recognition, leading to both primary and acquired resistance to $P D-1$ blockade in some cancer patients [37, 38]. Preclinical studies have suggested that targeting JAK/STAT3 could effectively suppress ovarian tumor progression and have therapeutic potential for treating advanced EOC [39-41]. Our observations suggest that although cancer cells may evade immunological attack by mutating genes such as JAK1, de novo T-cell responses against 
such mutations might be exploited to inhibit tumor growth in cancer patients. Interestingly, we discovered IL27RA mutations in 3 patients, including two loss-offunction truncating mutations and one missense mutation. The IL27RA missense mutation was shown to elicit concomitant $\mathrm{CD}^{+}{ }^{+}$and $\mathrm{CD}^{+}{ }^{+}$-cell responses. IL27RA is the alpha subunit of Interleukin 27 (IL27) receptor, which has been reported to demonstrate a dual role of anti-tumor activity and immune regulatory function [42, 43]. A recent proteomic analysis on human ovarian cancer cell lines revealed that $I L-27$ and $I F N-\gamma$ shared a broad set of activities, such as the HLA class I antigen presentation [44]. Loss of the IFN- $\gamma$ pathway gene has been shown as a mechanism of resistance to antiCTLA-4 therapy [45]. It is possible that mutations in IL27RA provide a novel immune-evasion mechanisms in EOC. Further validations and functional studies will be needed to determine the prognostic and therapeutic potential of IL27-IL27R pathway genes in EOC.

Our study reveals that a significant portion of neoantigen candidates can fail to pass the predefined criteria for mutant allele expression in RNAseq, and we showed some of them could elicit strong mutant-specific $\mathrm{CD} 4^{+}$ and/or $\mathrm{CD} 8^{+} \mathrm{T}$-cell response in autologous PBMCs and/ or TILs. Conceptually, the level of expression of the source protein is a surrogate for determining whether it reaches the threshold required for its efficient processing and presentation in HLA, and whether neoantigenspecific T-cells would efficiently reciognize the tumor target. The latter is mainly determined by affinity of TCR to interact with MHC/neoepitope complex. For virus antigen-specific T-cells, only a single $\mathrm{MHC}$ /peptide is sufficient to activate $\mathrm{T}$-cells through high-affinity TCRs [46]. Because affinity for neoantigen-specific TCRs is considered to be high due to absence of central tolerance, it is possible that low level of expression is enough to induce neoantigen-specific $\mathrm{T}$-cell responses. There have been multiple strategies to use expression information for neoantigen prediction, including studies using RNAseq from autologous tumor samples [8], studies using RNAseq data from different patients of the same cancer type (i.e., TCGA) [47], and studies not using expression information [22, 28, 48]. Even for the strategy of using RNAseq from autologous tumor samples, the criteria used by different studies to determine neoantigen expression can be vastly different. Therefore, it will be necessary in the future to conduct comprehensive assessments of the role of expression information and the approaches of leveraging it for neoantigen prediction and/or prioritization.

The nature of relatively low mutation burden of EOC also calls for culture enrichment approaches to expand neoepitope specific clones. In the study by Bobisse et al [22], whole tumors were cultured with high concentration of IL-2 in the presence MHC class I neoepitope pools [22]. In our study, we stimulated isolated $\mathrm{CD}^{+}$and $\mathrm{CD}^{+}$T-cells from tumors with peptide-pulsed $\mathrm{CD}^{-} \mathrm{CD}^{-}$cells derived from PBMCs, because APCs in ovarian tumor microenvironment have been shown to be dysfunctional or immunosuppressive [49]. A recent study by Yossef et al showed that the detection of neoantigen-reactive TILs could be enhanced by enriching T-cells that express PD-1 and/or T-cell activation markers followed by microwell culturing to avoid overgrowth of nonreactive T-cells [50]. As the availability of patients' specimens such as TILs is often limited, future studies are warranted to benchmark the prediction accuracy and effectiveness of different culture enrichment approaches for screening of immunogenic neoantigens in EOC.

We were able to isolate and clone TCRs from in vitro expanded $\mathrm{CD}^{+}{ }^{+}$and $\mathrm{CD} 8^{+} \mathrm{T}$-cell clones reactive against neoepitopes, and demonstrated the feasibility of specifically targeting the neoantigens by TCR gene-engineered T-cells. Our current protocoal takes about 2 weeks to prepare TCR-transduced T-cells from the established neoantigen-specific T-cell lines. Adoptive transfer of autologous tumor antigen-specific T-cells is an effective therapeutic treatment for cancer patients [51, 52]. Using tumor antigen-specific TCR genes, tumor antigenspecific T-cells can be rapidly expanded for infusion into patients in order to mediate immediate elimination of tumors and long-term tumor immune-surveillance. The therapeutic effects of ACT using TCR gene-engineered T-cells have been demonstrated in many clinical trials. High-affinity TCR gene is required to engineer effective $\mathrm{T}$-cells for strong recognition and destruction of cancer cells. It has been recently reported that function of neoepitope-specific $\mathrm{CD}^{+}$T-cell from ovarian tumors is significantly higher than PBMCs [22], suggesting neoepitope-specific $\mathrm{CD}_{4}^{+}$and/or $\mathrm{CD} 8^{+}$T-cells in TILs could be a useful source of TCR for ACT.

The neoepitope-specific TCRs could be introduced into naïve or central memory $\mathrm{T}$-cells to target driver mutations [11], simultaneously target multiple mutations, and combine ACT with other immunomodulators such as checkpoint inhibitors. Conceptually multiple neoantigens can be targeted but it is difficult to define an optimal number of neoepitopes. The range of neoepitopes utilized in published clinical trials of neoantigen vaccines varies between 7 and $20[8,9,53]$. Due to the intratumoral heterogeneity, it would be desirable to target multiple neoantigens for each patient. Although most previous ACT trials using TCR gene-engineered T cells targeted a single tumor antigen epitope, it is feasible to target multiple tumor antigens using a mixture of engineered T-cell products. As assessment of the immunosuppressive microenvironment was not the main 
focus of our current study, we did not include exogenous checkpoint blockade (e.g. anti-CTLA4 or anti-PD1) into our neoantigen recognition assays. It is well accepted that immunosuppression within the tumor microenvironment represents a critical barrier for antitumor $\mathrm{T}$ cell function, both neoantigen-reactive and adoptively transferred engineered $\mathrm{T}$ cells. A published report from a recent clinical trial evaluating adoptive transfer of TCR-engineered $\mathrm{T}$ cells and ipilimumab (anti-CTLA4) demonstrated that while addition of ipilimumab was safe and tolerable, there was no apparent clinical benefit from the combination [54]. Although the small sample size $(n=4)$ was insufficiently powered, this study and other preclinical/clinical studies suggest immunomodulatory combinations (e.g. anti-PD1, antiTGF- $\beta$, IDO inhibitors) could still potentiate therapeutic effect of ACT. Additional studies are required to empirically determine the optimal combination for eliciting clinical benefit. Our current study focused on analyzing treatment-naive ovarian cancer patients at the time of primary debulking surgery. Future studies will be warranted to determine intrinsic factors of tumor and microenvironment as well as the nature of TCR affecting the infiltration of adoptively transferred T cells. In future ACT clinical trials using TCR gene-engineered T cells, it is also feasible to quantify engineered T cells in the tumors using quantitative TCR or digital PCR. Taken together, these results warranted future studies of patientspecific neoantigens as potential targets for downstream translational validation towards adoptive T-cell immunotherapy of ovarian cancer.

\section{Conclusion}

In summary, our study demonstrated the feasibility of efficiently identifying both $\mathrm{CD}^{+}$and $\mathrm{CD}^{+}$neoantigenspecific T-cells in ovarian cancer. Further methodology development [55], including the improvement of patient response prediction, neoantigen prediction and prioritization, T-cell enrichment and TCR engineering, will be warranted to exploit the therapeutic potential of neoantigen-targeting for personalized immunotherapy in treating advanced EOC.

\section{Methods}

\section{Patient and sample characteristics}

Tumor specimens were collected at the time of primary debulking surgery, flash frozen in liquid nitrogen and stored at $-80^{\circ} \mathrm{C}$. Portions of tumor specimens were dissociated by the Gentle MACS system (Miltenyi Biotec) to obtain single-cell suspensions. After a density gradient isolation, live mononuclear cells were stored in liquid nitrogen until use. PBMC were obtained using a density gradient method and stored in liquid nitrogen. DNA was extracted from the frozen tissues and PBMCs using the GenFIND DNA extraction kit (Agencourt, Pasadena, CA) per the manufacturer's instructions. All pathology specimens were reviewed by experienced gynecologic pathologists and tumors were classified according to the WHO criteria [56]. Prior to surgery, no patients received neo-adjuvant chemotherapy, and subsequent to surgery all patients received adjuvant platinum/ taxane-based chemotherapy. No patient had received immunotherapy treatment prior to surgery.

\section{Whole-exome sequencing and somatic mutation calling}

Individual exome capture of each DNA sample followed by single-indexed library generation was carried out using the SureSelect XT Target Enrichment System (Human All Exon V5 kit, Agilent Inc). Cluster generation on cBot was followed by $2 \times 100$ bp pairedend (PE) sequencing on a HiSeq2500 sequencer according to the manufacturer's recommended protocol (Illumina Inc.). High quality WES paired-end reads passing Illumina RTA filter were aligned to the NCBI human reference genome (GRCh37) using BurrowsWheeler Alignment (BWA) tool [57]. PCR duplicated reads were marked and removed using Picard tool. All samples had more than $80 \%$ of the targeted regions covered by at least 30-fold. Putative mutations were identified by running variation detection module of Bambino [58] and Strelka [59], and then further filtered as previously described $[25,60]$. All putative singlenucleotide variants (SNVs) were combined and further filtered based on a standard set of criteria to remove the following common types of false calls: [1] the alternative allele is present in the matching normal sample and the contingency between the tumor and normal samples is not statistically significant; [2] the mutant alleles are only present in one strand and the strand bias is statistically significant; [3] the putative mutation occurs at a site with systematically dropped base quality scores; [4] the reads harboring the mutant allele are associated with poor mapping quality. The identified somatic mutations were compared to the public human germline databases including dbSNP [61], 1000 Genomes Project [62], NHLBI's Exome Sequencing Project to further exclude remaining germline polymorphisms. All mutations were manually reviewed to ensure accuracy and annotated using ANNOVAR [63] with the latest NCBI RefSeq database. To extract somatic copy number information based on the sequenced exomes of the samples, Varscan2 [64] was employed, and the results were smoothed and segmented with the Bioconductor DNACopy package [65]. GISTIC2.0 [66] was used to identify recurrent somatic chromosomal alterations in the five samples with mutant-specific T-cell response and the five samples without, respectively. 


\section{RNAseq and gene expression analysis}

RNAs were purified from fresh frozen tumors using the miRNeasy mini kit (Qiagen). The sequencing libraries were prepared with the TruSeq Stranded Total RNA kit (Illumina Inc) and sequenced for 100 cycle paired-end on a HiSeq2500 sequencer, following the manufacturer's recommended protocol (Illumina Inc.). Raw reads from RNAseq that passed the Illumina RTA quality filter were first pre-processed using Cutadapt to remove adapter sequences, followed by FASTQC for sequencing base quality control. The remaining reads were mapped to the NCBI human reference genome (GRCh37) and RefSeq annotation database using Tophat [67]. A second round of quality control was performed to identify potential RNAseq library preparation problems by examining mapped BAM files using RSeQC [68]. From the mapping results, the number of reads aligning to each gene was calculated using HTSeq [69] and RPKM (Reads Per Kilobase of transcript per Million mapped reads) values were obtained using RSEM [70]. ssGSEA [71], an extension of Gene Set Enrichment Analysis [72], was performed on gene expression data of each sample using the Reactome gene sets obtained from the MSigDB database [73]. The normalized enrichment score of each gene set in each sample was retrieved, and compared between the five samples with mutant-specific T-cell response and the five samples without. Differentially expressed genes (DEGs) between those patients who responded and those who didn't were determined using LIMMA [74]. By intersecting the list of DEGs $(p<0.05)$ with the genes involved in the antigen processing and presentation machinery (APPM) pathways, a signature of APPM constsiting of 31 DEGs were obtained. TCGA gene expression dataset were downloaded from cBioportlal [75] using Ovarian Serous Cystadenocarcinoma (TCGA Provisional, 307 samples with RNAseq). TCGA ovarian cancer patients were ranked based on median expression value of the 31-gene signature and grouped in top vs bottom tertile (groups of high versus low expression level of signature). Survival analysis was done using the survival package in R. Precalculated dataset of tumor-infiltrating subtypes for the TCGA Ovarian cancer patients were downloaded from xCell [76] and the comparsion of tumor-infiltrating subtypes between groups of high versus low expression level of signature was performed using Student's t-test. Correlation between the expression of APPM signature and $M Y C$ was calculated using Pearson correlation, and a linear model was built to fit the data and test significance and was plotted as trendline with the confidence intervals.

\section{Neoantigen prediction}

For each missense SNVs, we obtained 8 to 15-mer peptide sequences containing the mutated amino acid as well as corresponding wild-type ones from RefSeq [77]. Genotypes for patients' class I and II HLA alleles were determined from next-generation sequencing data using Polysolver and HLAminer, respectively, with default parameters [78, 79]. Class I and II-binding affinities for each combination of peptide/HLA type were predicted using NetMHCpan v3.0 [80-82] and NetMHCIIpan v3.1 [80], respectively, with default parameters. For a mutant peptide to be considered as a neoepitope, we required: 1) mutant binding affinity score is less than $150 \mathrm{nM}$; 2) the ratio of binding affinity between mutant and the matched wild-type peptides is less than 0.9; and 3) the difference in binding affinity between mutant and wildtype epitopes is at least $100 \mathrm{nM}$, except for peptides from any Cancer Census genes [26]. If one mutation was predicted to generate multiple neoepitopes, it was counted as one neoantigen [48]. For the predicted neoantigen, we assessed the expression of mutant allele in RNAseq using a set of previously published criteria for neoantigen expression [83]: 1) at least two supporting reads in RNAseq; 2) minimum variant allele fraction (VAF) of $4 \%$ for mutations with at least three reads or $20 \%$ for mutations with exact two supporting reads; 3 ) no significant strand bias $(p<0.05)$.

\section{Peptide synthesis}

Synthetic peptides for neoepitopes and the corresponding wild-type epitopes were manufactured at $>90 \%$ purity (Genscript, Piscataway, NJ). Reverse phase HPLC produced lyophilized peptides were reconstituted in DMSO (Sigma) and used to test T-cell reactivity.

\section{Monitoring of neoepitope-specific T-cell response}

Mutated peptide specific T-cell response was investigated using T-cell presensitization method as previously described [84]. Briefly, $\mathrm{CD}^{+}$and $\mathrm{CD}^{+}{ }^{+}$T-cells were sequentially isolated from PBMCs or tumors of EOC patients using Dynabeads CD8 and CD4 positive isolation kits (ThermoFisher Scientific) and incubated overnight in a $37{ }^{\circ} \mathrm{C} 5 \% \mathrm{CO}_{2}$ incubator. Remaining $\mathrm{CD}^{-} \mathrm{CD}^{-}$cells from PBMCs were used as APCs for pulsing a pool of $2 \mu \mathrm{M}$ patient-specific mutated peptides overnight, irradiated at $3000 \mathrm{rad}$, washed and mixed with $\mathrm{CD}^{+}{ }^{+}$or $\mathrm{CD}^{+}{ }^{+}$-cells. The $\mathrm{CD}^{+}$and $\mathrm{CD}^{+} \mathrm{T}$-cells were cultured in the presence of $10 \mathrm{U} / \mathrm{ml} \mathrm{IL-2} \mathrm{(Roche)} \mathrm{and}$ $10 \mathrm{ng} / \mathrm{ml} \mathrm{IL-7} \mathrm{(R \& D} \mathrm{systems).} \mathrm{A} \mathrm{part} \mathrm{of} \mathrm{CD4}{ }^{+}$T-cells were polyclonally activated by phytohemagglutinin (PHA, Remel-ThermoFisher Scientific) and cultured in the presence of IL-2 and IL-7 to prepare T-APC (antigen-presenting T-cells) [85]. At 13-15 days after the culture, these $\mathrm{CD}_{4}^{+}$and $\mathrm{CD}^{+}{ }^{+}$T-cells were harvested and tested reactivity against individual mutated or wild-type peptides, or pooled peptides-loaded on autologous T-APC by IFN- $\gamma$ ELISPOT assay as previously described [84]. 
Neoepitope-specific response was considered as positive with a minimum of 25 IFN- $\gamma$-spot-forming cells against mutated peptide per $5 \times 10^{4}$ cells as well as the number of spots was 2-fold higher than the corresponding wild-type peptide [9].

\section{Establishment of neoepitope-specific T-cell clones}

In order to characterize in details neoantigen recognition by neoepitope-specific T-cells, we established neoepitopespecific T-cell clones. For $\mathrm{CD}^{+}{ }^{+} \mathrm{T}$-cells, presensitized Tcells that showed neoantigen-specific reactivity in ELISPOT assays were restimulated by mutated peptide-pulsed T-APC and IFN- $\gamma$-producing T-cells were labeled using IFN- $\gamma$-capture reagent (Miltenyi Biotec) and sorted by flow-cytometry as described [86]. For $\mathrm{CD}^{+}{ }^{+} \mathrm{T}$-cells, presensitized T-cells were similarly restimulated in the presence of monensin (Sigma) and phycoerythrin (PE)-labeled anti-CD154 monoclonal antibody (mAb) as described [87] and CD154expressing cells were sorted. Isolated $\mathrm{T}$-cells were polyclonally expanded by PHA stimulation in the presence of allogeneic irradiated PBMC, IL-2 and IL-7. Purity and clonality of T-cells were tested by lowresolution TCR spectratyping using V $\beta$ subtypespecific antibodies (Beckman Coulter). For some oligoclonal $T$-cell cultures containing different $V \beta$ expressing T-cells, cells were sorted again based on $V \beta$ expression. Reactivity of neoepitope-specific T-cell clones were tested by ELISA and/or intracellular cytokine staining [88].

\section{Engineering neoepitope-specific T-cells by TCR gene transduction}

Retroviral vectors expressing neoepitope-specific TCR genes were constructed as described previously with modifications [32]. Briefly, a part of sorted neoepitopespecific T-cells (2,000-5,000 T-cells) were lysed in TRIReagent. Total RNA was extracted by columns (Zymo Research), and reverse transcribed using SuperScript IV First-Strand Synthesis System (Thermo Fisher Scientific) using oligo dT primers according to the manufacturer's instruction. Variable regions for TCR $\alpha$ and $\beta$ chains were independently PCR-amplified using multiplexed primers, and assembled into a retroviral plasmid vector together with constant regions. Plasmids were amplified in NEBStable competent E. coli (New England Biolabs) and extracted using columns (ZymoResearch). To generate retroviral particles, GP2-293 packaging cell line (Clontech) was co-transfected with TCR-expressing transfer plasmid and envelope (pVSV-G: Clontech) plasmid using Lipofectamine 3000 reagent (Thermo Fisher Scientific). Medium was exchanged $7 \mathrm{~h}$ after transfection. Retroviral vectors were harvested at around 36 and $60 \mathrm{~h}$ after changing medium. PBMCs from healthy individuals were activated by PHA in the presence of IL-2, IL-7 and $10 \mathrm{ng} / \mathrm{ml}$ IL-12 (Peprotech). Activated T-cells were harvested at 36-48 h, and transduced by $125 \mu \mathrm{l}$ freshly harvested retroviral vectors in a 96-well flat-bottom plate which was coated with Retronectin (TaKaRa Bio) and anti-CD3 mAb (OKT3; eBioscience). Transduction was repeated at $24 \mathrm{~h}$ after the first transduction on Retronectin without anti-CD3 $\mathrm{mAb}$. In some experiments, we established PG13 clones stably producing high-titer retroviral vectors as described [89]. Briefly, PG13 (ATCC) was transduced with retroviral supernatant from GP2-293 and cloned by limiting dilution. To infect Tcells with PG13-derived retroviral particles, retroviral supernatant was added in a 96-well plate which was coated with Retronectin with or without anti-CD3 mAb and incubated for $6 \mathrm{~h}$. After removing retroviral supernatant, the plate was rinsed by PBS containing $1 \%$ bovine serum albumin (BSA). Activated T-cells were transduced as described above. Function of transduced T-cells was investigated 3-7 days after the second transduction.

\section{Statistics}

Statistical analysis was performed using R. Primary statistical tests include Student's t test for single comparisons of normally distributed data, Chi-square test for comparsion of BRCA1/2 mutation rate, Pearson's correlation test for comparisons of APPM signature expression versus MYC expression, and Cox ProportionalHazards Model to compare survival of TCGA patients with high versus low expression of APPM signature. All statistical tests are 2-tailed unless otherwise specified. A $p$-value less than 0.05 was considered significant.

\section{Additional files}

Additional file 1: Figure S1. The protein view of selected genes with somatic mutations in the 20 EOC patients studied. (a) TP53. (b) BRCA1. (c) BRCA2. (d) IL27RA. Note that splicing site mutations (c.673-2A > G in TP53 and C.4987-1G > T in BRAC1) are not shown. (PPTX $130 \mathrm{~kb}$ )

Additional file 2: Figure S2. Comparison of somatic mutation and neoantigen between primary and local invasive tumors. (a) Somatic mutation. (b) Neoantigen. Pt \#02 on the left and Pt \#10 on the right. Xaxis: variant allele frequency (VAF) in WES of primary tumor. Y-axis: VAF in WES of local invasive tumor. (PPTX $260 \mathrm{~kb}$ )

Additional file 3: Figure S3. Spontaneous $\mathrm{CD}^{+} \mathrm{T}$-cell response against mutant-specific epitopes from autologous tumor-infiltrating lymphocytes (TIL) and/or peripheral blood lymphocytes (PBL). T-cell reactivity was measured by IFN- $\gamma$ ELISpot assay (Methods). (-): no peptide; Wt: wildtype peptide; Mt.: Mutant peptide. (PPTX $85 \mathrm{~kb}$ )

Additional file 4: Figure S4. Spontaneous $C D 8^{+} T$-cell response against mutant-specific epitopes from autologous tumor-infiltrating lymphocytes (TIL) and/or peripheral blood lymphocytes (PBL). T-cell reactivity was measured by IFN- $\gamma$ ELISpot assay (Methods). (-): no peptide; Wt: wildtype peptide; Mt.: Mutant peptide. (PPTX $84 \mathrm{~kb}$ )

Additional file 5: Figure S5. The expression level of a panel of 10 immune inhibitory molecules from the tumor RNA-Seq data. These 
immune inhibitory molecules include PD1, PDL1, CTLA4, CD80, CD86, LAG3, TIM3, LAGLS9, MYC and FOXP3. The patients shown here include Pt \#2, Pt \#8, Pt \#16 and Pt \#5. (PPTX 78 kb)

Additional file 6: Figure S6. Recurrent somatic copy number alternations by the GISTC2.0 algorithm. GISTIC deletion (left) and amplification (right) plots using data from the five patients with mutantspecific T-cell response (top), and data from the five patients without mutant-specific T-cell response (bottom). The genome is oriented vertically from top to bottom, and GISTIC q-values at each locus are plotted from left to right on a log scale. The green line represents the default significance threshold (q-value $=0.25$ ). For each plot, known or interesting cancer genes are highlighted. (PPTX $278 \mathrm{~kb}$ )

Additional file 7: Figure S7. TCR V $\beta$ usage of NUP214 neopeptidespecific CD4 ${ }^{+}$T-cell line. (a) TCR V $\beta$ usage of NUP214 neopeptide-specific $C D 4^{+}$T-cell line was determined by flow cytometry. (b) NUP214 neopeptide-specific IFN- $\gamma$ production from $\mathrm{V} \beta 2^{+}, \mathrm{V} \beta 13.1^{+}$, or $\mathrm{V} \beta 2^{-} \mathrm{V} \beta 13.1^{-}$ T-cells was examined by intracellular cytokine staining. (PPTX $221 \mathrm{~kb}$ )

Additional file 8: Figure S8. Characterization of JAK1 neoepitopespecific $\mathrm{CD}^{+}{ }^{+}$T-cells. (a) Peptide reactivity of a JAK1 neoepitope-specific $\mathrm{CD}^{+} \mathrm{T}$-cell line. IFN- $\gamma$ and GM-CSF production on $\mathrm{CD}^{+}{ }^{+} \mathrm{T}$-cells against JAK1 mutated (IEILRNLYHEIIV) or wild-type (IEILRNLYHENIV) peptidepulsed autologous EBV-B-cells were determined by intracellular cytokine staining. (b) TCR usage of JAK1 neoepitope-specific $C D 4^{+} \mathrm{T}$-cell line. Tcells were stained with TCR V $\beta$ subtype-specific antibodies and analyzed by flow cytometry. (c) Purity of $\mathrm{V} \beta 13.6^{+}$cells after magnetic-beads sorting. (d) Avidity of JAK1 neoepitope-specific T-cell clone. CD4 ${ }^{+}$T-cell clones were stimulated with autologous EBV-B-cells pulsed with the indicated concentration of mutated or wild-type peptide for $6 \mathrm{~h}$ in the presence of Golgi stop. IFN- $\gamma$ production from $V \beta 13.6^{+}$cells were determined by flow cytometry. The data represents mean \pm s.d. of duplicate wells. (e) Recognition of autologous tumor-derived cells by V $313.6^{+}$T-cell clone. PBMC or AMC were co-cultured with VB13.6 JAK1 neoepitope-specific CD4 ${ }^{+} \mathrm{T}$ clones or without T-cells (-) for $24 \mathrm{~h}$. AMC: ascites-derived mononuclear cells. IFN- $\gamma$ production was measured by ELISA. The data represent mean + s.d. of duplicate wells. ${ }^{*} p<0.05$ compared to IFN- $\gamma$ level against PBMC. (PPTX $250 \mathrm{~kb}$ )

Additional file 9: Figure S9. Reactivity of NUP214 neoepitope-specific TCR-transduced $\mathrm{CD}^{+}$and $\mathrm{CD} 4^{+}$T-cells. IFN- $\gamma$ production (a) and GM-CSF (b) production from $\mathrm{V} \beta 13.1^{+}$or $\mathrm{V} \beta 2^{-} \mathrm{V} \beta 13.1^{-} \mathrm{TCR}$ - transduced $\mathrm{CD} 8^{+}$and CD8 ${ }^{-}\left(C D 4^{+}\right)$T-cells against EBV-B-cells pulsed with or without NUP214 mutated or wild-type peptide were determined by intracellular cytokine staining. (PPTX $683 \mathrm{~kb}$ )

Additional file 10: Figure S10. Analysis of TRPC4 neoepitope-specific CD8 ${ }^{+}$T-cell clone. (a) IFN- $\gamma$ and GM-CSF production on CD8 ${ }^{+}$T-cells against TRPC4 mutated (QSLFWSIFV) or wild-type (QSLFWSIFG) peptidepulsed autologous EBV-B-cells were determined by intracellular cytokine staining. (b) Avidity of TRPC4 neoepitope-specific T-cell clone. CD8 ${ }^{+}$T-cell clone was co-cultured with autologous EBV-B-cells pulsed with the different concentration of mutated or wild-type peptide for $24 \mathrm{~h}$. IFN- $\gamma$ level in the culture supernatant was measured by ELISA. The data represents mean \pm s.d. of duplicate wells. (c) IFN- $\gamma$ production from TRPC4 neoepitope-specific TCR-transduced T-cells against EBV-B pulsed with or without TRPC4 mutated or wild-type peptide was determined by intracellular cytokine staining. (PPTX $321 \mathrm{~kb}$ )

Additional file 11: Figure S11. The expression level of APPM signature from the tumor RNA-Seq data. The APPM expression is derived from the median expression value (in RPKM) of the 31 genes within the APPM signature (Methods). The patients shown here include Pt \#3, Pt \#15, Pt \#19 and Pt \#5. (PPTX $50 \mathrm{~kb}$ )

Additional file 12: Table S1. Clinical demographics of treatment-naïve (including both chemotherapy and immunotherapy) epithelial ovarian cancer patients at the time of primary debulking surgery. Patient identification number, age at diagnosis, tumor histologic type, FIGO stage, debulking status, residual tumor mass after debulking surgery, number of recurrences after primary debulking surgery, and RECIST to frontline chemotherapy after primary debulking surgery. (XLSX $10 \mathrm{~kb}$ )

Additional file 13: Table S2. Somatic point mutations identified from whole-exome sequencing. (a) The 18 patients with either primary tumor or locally invasive tumor; (b) The 2 patients with both primary tumor and locally invasive tumor. AA, amino acid; CGC, cancer gene census; VAF, variant allele frequency. (XLSX $244 \mathrm{~kb}$ )

Additional file 14: Table S3. Somatic mutation burdens and predicted neoantigen load in the 20 patients. The predicted neoantigens are classified as expressed or non-expressed based on the mutant allele's expression level in RNAseq data (see Method section). (XLSX $10 \mathrm{~kb}$ )

Additional file 15: Table S4. Description of the 75 neopeptides screened for immunogenicity. The expression status is based on the mutant allele's expression level in RNAseq data, and affinity score is predicted by NetMHC algorithm with default setting (see Method section). (XLSX $15 \mathrm{~kb}$ )

Additional file 16: Table S5. The list of 31 genes within the derived APPM signature (see Method section). (XLSX $11 \mathrm{~kb}$ )

\section{Abbreviations}

AA: Amino acid; ACT: Adoptive cell therapy; AMC: Ascites mononuclear cells; APC: Antigen presenting cells; APPM: Antigen processing and presentation machinery; BSA: Bovine serum albumin; CGC: Cancer gene census; CLP: Christmas light plot; DEG: Differentially expressed genes; DMSO: Dimethyl sulfoxide; EBV: Epstein-Barr virus; ELISpot: Enzyme-linked immune absorbent spot; EOC: Epithelial ovarian cancer; FIGO: International Federation of Gynaecology and Obstetrics; GM-CSF: Granulocyte macrophage colony stimulating factor; HLA: Human leukocyte antigen; HPLC: High-performance liquid chromatography; IFN: Interferon; IL: Interleukin; mAb: Monoclonal antibody; MHC: Major histocompatibility complex; PBL: Peripheral blood lymphocytes; PBMC: Peripheral blood mononuclear cells; PBS: Phosphate buffered saline; Pt\#: Patient number; RECIST: Response Evaluation Criteria In Solid Tumors; RNAseq: RNA sequencing; s.d.: standard deviation; SNV: Single nucleotide variants; TCGA: The Cancer Genome Atlas; TCR: T-cell receptor; TLL: Tumor infiltrating lymphocytes; TMC: Tumor mononuclear cells; VAF: Variant allele frequency; WES: Whole exome sequencing

\section{Acknowledgements}

We thank Kristen Starbuck, MD for material support.

\section{Authors' contributions}

$\mathrm{SL}, \mathrm{JM}$, and $\mathrm{KOO}$ conceived the study and served as the primary author of the paper. SL, JM, LW, TT, SB, AAL, and KOO planned, performed, and analyzed the majority of the studies, and prepared the manuscript and Figs. LW developed the neoantigen prediction and prioritization pipelines. JM and TT performed the functional experiments. AM, SBL, and AAL coordinated samples and clinical data. SL, JM, LW, TT, SB, QH, EC, LW, LY, LM, NWB, SY, QZ, TPC, JW, GLM, AAL, and KOO analyzed and interpreted data. All authors read and approved the final manuscript.

\section{Funding}

This work was supported by the RPCI-UPCI Ovarian Cancer SPORE P50 CA159981, NCl Cancer Center Support Grant P30 CA016056, NIH U24 CA232979, NIH R01 CA158318, the Empire State Stem Cell Board from the New York State Health Department (NYSTEM) N14C-002, Ovarian Cancer Research Alliance, and the Roswell Park Alliance Foundation.

\section{Availability of data and materials}

The datasets generated and/or analyzed during the current study are available from the corresponding author on reasonable request.

\section{Ethics approval and consent to participate}

Biospecimens of epithelial ovarian cancer patients were obtained with informed consent at Roswell Park Comprehensive Cancer Center in accordance with a protocol approved by the institutional review board.

\section{Consent for publication}

Not applicable.

Competing interests

The authors declare that they have no competing interests. 


\section{Author details}

'Department of Biostatistics and Bioinformatics, Roswell Park Comprehensive Cancer Center, Buffalo, NY 14263, USA. ${ }^{2}$ Center for Immunotherapy, Roswell Park Comprehensive Cancer Center, Buffalo, NY 14263, USA. ${ }^{3}$ Gynecologic Cancer Center of Excellence, Department of Obstetrics and Gynecology, John P. Murtha Cancer Center, Uniformed Services University and Walter Reed National Military Medical Center, Bethesda, MD 20889, USA. ${ }^{4}$ Department of Gynecologic Oncology, Roswell Park Comprehensive Cancer Center, Buffalo, NY 14263, USA. ${ }^{5}$ Department of Cancer Prevention and Control, Roswell Park Comprehensive Cancer Center, Buffalo, NY 14263, USA. ${ }^{6}$ Department of Obstetrics and Gynecology, Inova Fairfax Medical Campus, Falls Church, VA 22003, USA. ${ }^{7}$ Inova Schar Cancer Institute, Falls Church, VA 22003, USA.

\section{Received: 12 March 2019 Accepted: 30 May 2019}

\section{Published online: 20 June 2019}

\section{References}

1. Siegel Rebecca L, Miller Kimberly D, Jemal A. Cancer statistics, 2016. CA Cancer J Clin. 2016:66:7-30.

2. Curiel TJ, et al. Specific recruitment of regulatory $T$ cells in ovarian carcinoma fosters immune privilege and predicts reduced survival. Nat Med. 2004;10:942-9.

3. Matsuzaki J, et al. Tumor-infiltrating NY-ESO-1-specific CD8+ T cells are negatively regulated by LAG-3 and PD-1 in human ovarian cancer. Proc Nat Acad Sci U S A. 2010;107:7875-80.

4. Sato $E$, et al. Intraepithelial CD8+ tumor-infiltrating lymphocytes and a high CD8+/regulatory $T$ cell ratio are associated with favorable prognosis in ovarian cancer. Proc Natl Acad Sci U S A. 2005;102: 18538-43.

5. Zhang $L$, et al. Intratumoral T cells, recurrence, and survival in epithelial ovarian cancer. N Engl J Med. 2003;348:203-13.

6. Schumacher TN, Schreiber RD. Neoantigens in cancer immunotherapy. Science. 2015;348:69.

7. Snyder A, et al. Genetic basis for clinical response to CTLA-4 blockade in melanoma. N Engl J Med. 2014;371:2189-99.

8. Ott PA, et al. An immunogenic personal neoantigen vaccine for patients with melanoma. Nature. 2017;547:217-21.

9. Sahin $U$, et al. Personalized RNA mutanome vaccines mobilize poly-specific therapeutic immunity against cancer. Nature. 2017;547:222-6.

10. Tran $\mathrm{E}$, et al. Cancer immunotherapy based on mutation-specific CD4+ T cells in a patient with epithelial cancer. Science. 2014;344:641-5.

11. Tran $\mathrm{E}$, et al. T-cell transfer therapy targeting mutant KRAS in Cancer. N Engl J Med. 2016:375:2255-62

12. Zsiros E, Tanyi J, Balint K, Kandalaft LE. Immunotherapy for ovarian cancer: recent advances and perspectives. Curr Opin Oncol. 2014:26:492-500.

13. van Rooij N, et al. Tumor exome analysis reveals neoantigen-specific T-cell reactivity in an ipilimumab-responsive melanoma. J Clin Oncol. 2013:31: e439-42.

14. Lu YC, et al. Efficient identification of mutated cancer antigens recognized by $T$ cells associated with durable tumor regressions. Clin Cancer Res. 2014; 20:3401-10

15. Carreno BM, et al. Cancer immunotherapy A dendritic cell vaccine increases the breadth and diversity of melanoma neoantigen-specific T cells. Science. 2015;348:803-8

16. Robbins PF, et al. Mining exomic sequencing data to identify mutated antigens recognized by adoptively transferred tumor-reactive T cells. Nat Med. 2013;19:747-52

17. Linnemann C, et al. High-throughput epitope discovery reveals frequent recognition of neo-antigens by CD4+ T cells in human melanoma. Nat Med. 2015:21:81-5.

18. Kreiter $\mathrm{S}$, et al. Mutant MHC class II epitopes drive therapeutic immune responses to cancer. Nature. 2015;520:692-6.

19. McGranahan $\mathrm{N}$, et al. Clonal neoantigens elicit $\mathrm{T}$ cell immunoreactivity and sensitivity to immune checkpoint blockade. Science. 2016;351:1463-9.

20. Wick DA, et al. Surveillance of the tumor mutanome by $T$ cells during progression from primary to recurrent ovarian cancer. Clin Cancer Res. 2014; 20:1125-34.

21. Martin SD, et al. Low mutation burden in ovarian Cancer may limit the utility of Neoantigen-targeted vaccines. PLoS One. 2016;11:e0155189.
22. Bobisse $\mathrm{S}$, et al. Sensitive and frequent identification of high avidity neoepitope specific CD8 (+) T cells in immunotherapy-naive ovarian cancer. Nat Commun. 2018;9:1092

23. Deniger DC, et al. T-cell responses to TP53 "hotspot" mutations and unique Neoantigens expressed by human ovarian cancers. Clin Cancer Res. 2018;24: 5562-73.

24. Wei $L$, et al. Intratumoral and Intertumoral genomic heterogeneity of multifocal localized prostate Cancer impacts molecular classifications and genomic prognosticators. Eur Urol. 2016.

25. Wei $\mathrm{L}$, et al. Whole-genome sequencing of a malignant granular cell tumor with metabolic response to pazopanib. Molecular Case Studies. 2015;1.

26. Futreal PA, et al. A census of human cancer genes. Nat Rev Cancer. 2004;4: 177-83.

27. Duan F, et al. Genomic and bioinformatic profiling of mutational neoepitopes reveals new rules to predict anticancer immunogenicity. J Exp Med. 2014;211:2231-48.

28. Rajasagi $\mathrm{M}$, et al. Systematic identification of personal tumor-specific neoantigens in chronic lymphocytic leukemia. Blood. 2014;124:453-62.

29. Sykulev Y, Joo M, Vturina I, Tsomides TJ, Eisen HN. Evidence that a single peptide-MHC complex on a target cell can elicit a cytolytic T cell response. Immunity. 1996:4:565-71.

30. Casey SC, et al. MYC regulates the antitumor immune response through CD47 and PD-L1. Science. 2016:352:227-31.

31. Yoshihara K, et al. High-risk ovarian cancer based on 126-gene expression signature is uniquely characterized by downregulation of antigen presentation pathway. Clin Cancer Res. 2012;18:1374-85.

32. Tsuji T, et al. Rapid construction of antitumor T-cell receptor vectors from frozen tumors for engineered T-cell therapy. Cancer Immunol Res. 2018.

33. Ayyoub M, Pignon P, Classe JM, Odunsi K, Valmori D. CD4+ T effectors specific for the tumor antigen NY-ESO-1 are highly enriched at ovarian cancer sites and coexist with, but are distinct from, tumor-associated Treg. Cancer Immunol Res. 2013:1:303-8.

34. Tanyi $J$, et al. Personalized cancer vaccine effectively mobilizes antitumor $T$ cell immunity in ovarian cancer. Sci Transl Med. 2018;10.

35. Grimwade D, et al. Refinement of cytogenetic classification in acute myeloid leukemia: determination of prognostic significance of rare recurring chromosomal abnormalities among 5876 younger adult patients treated in the United Kingdom Medical Research Council trials. Blood. 2010;116:354-65.

36. Oancea C, Ruster B, Henschler R, Puccetti E, Ruthardt M. The t $(6 ; 9)$ associated DEK/CAN fusion protein targets a population of long-term repopulating hematopoietic stem cells for leukemogenic transformation. Leukemia. 2010;24:1910-9.

37. Shin, D.S. et al. Primary Resistance to PD-1 Blockade Mediated by \&lt;em\&gt; JAK1/2\&lt;/em\&gt; Mutations. Cancer Discovery 7, 188 (2017).

38. Zaretsky JM, et al. Mutations associated with acquired resistance to PD-1 blockade in melanoma. N Engl J Med. 2016;375:819-29.

39. Wen $W$, et al. Targeting JAK1/STAT3 signaling suppresses tumor progression and metastasis in a peritoneal model of human ovarian cancer. Mol Cancer Ther. 2014;13:3037-48.

40. Gritsina $\mathrm{G}$, et al. Targeted blockade of JAK/STAT3 signaling inhibits ovarian carcinoma growth. Mol Cancer Ther. 2015:14:1035.

41. Wen W, et al. Synergistic anti-tumor effect of combined inhibition of EGFR and JAK/STAT3 pathways in human ovarian cancer. Mol Cancer. 2015;14:100.

42. Di Carlo $\mathrm{E}_{\text {, et }}$ al. The antitumor potential of Interleukin-27 in prostate cancer Oncotarget. 2014;5:10332-41.

43. Dibra $D$, et al. Lack of Immunomodulatory Interleukin-27 enhances oncogenic properties of mutant p53 in vivo. Clin Cancer Res. 2016;22: 3876-83.

44. Petretto A, et al. Proteomic analysis uncovers common effects of IFN- $\gamma$ and IL-27 on the HLA class I antigen presentation machinery in human cancer cells. Oncotarget. 2016;7:72518-36.

45. Gao J, et al. Loss of IFN- $\gamma$ Pathway Genes in Tumor Cells as a Mechanism of Resistance to Anti-CTLA-4 Therapy. Cell. 2016:167:397-404.e9.

46. Irvine DJ, Purbhoo MA, Krogsgaard M, Davis MM. Direct observation of ligand recognition by T cells. Nature. 2002:419:845-9.

47. Anagnostou $V$, et al. Evolution of Neoantigen landscape during immune checkpoint blockade in non-small cell lung Cancer. Cancer Discov. 2017:7: 264-76.

48. Riaz N, et al. Tumor and Microenvironment Evolution during Immunotherapy with Nivolumab. Cell. 2017;171:934-949 e15. 
49. Wilke CM, Kryczek I, Zou W. Antigen-presenting cell (APC) subsets in ovarian cancer. Int Rev Immunol. 2011;30:120-6.

50. Yossef $R$, et al. Enhanced detection of neoantigen-reactive $T$ cells targeting unique and shared oncogenes for personalized cancer immunotherapy. $\mathrm{JCl}$ Insight. 2018;3

51. Rosenberg SA. Cell transfer immunotherapy for metastatic solid cancer-what clinicians need to know. Nat Rev Clin Oncol. 2011;8:577-85.

52. Yang $F$, et al. Adoptive cellular therapy (ACT) for Cancer treatment. Adv Exp Med Biol. 2016;909:169-239.

53. Keskin DB, et al. Neoantigen vaccine generates intratumoral T cell responses in phase Ib glioblastoma trial. Nature. 2019;565:234-9.

54. Nowicki TS, et al. A pilot trial of the combination of transgenic NY-ESO-1reactive adoptive cellular therapy with dendritic cell vaccination with or without Ipilimumab. Clin Cancer Res. 2019;25:2096-108.

55. Schoenberger SP. Is It Possible to Develop Cancer Vaccines to Neoantigens, What Are the Major Challenges, and How Can These Be Overcome? Targeting the Right Antigens in the Right Patients. Cold Spring Harb Perspect Biol. 2018;10.

56. Tavassoéli, F.A., Devilee, P. Pathology and Genetics of Tumours of the Breast and Female Genital Organs, (2003).

57. Li H, Durbin R. Fast and accurate short read alignment with burrowswheeler transform. Bioinformatics. 2009;25:1754-60.

58. Edmonson MN, et al. Bambino: a variant detector and alignment viewer for next-generation sequencing data in the SAM/BAM format. Bioinformatics. 2011;27:865-6.

59. Saunders $C T$, et al. Strelka: accurate somatic small-variant calling from sequenced tumor-normal sample pairs. Bioinformatics. 2012;28:1811-7.

60. Holmfeldt $\mathrm{L}$, et al. The genomic landscape of hypodiploid acute lymphoblastic leukemia. Nat Genet. 2013;45:242-52.

61. Sherry ST, et al. dbSNP: the NCBI database of genetic variation. Nucleic Acids Res. 2001;29:308-11.

62. Genomes Project C, et al. An integrated map of genetic variation from 1,092 human genomes. Nature. 2012;491:56-65.

63. Wang K, Li M, Hakonarson H. ANNOVAR: functional annotation of genetic variants from high-throughput sequencing data. Nucleic Acids Res. 2010;38: e164.

64. Koboldt DC, et al. VarScan 2: somatic mutation and copy number alteration discovery in cancer by exome sequencing. Genome Res. 2012:22:568-76.

65. Huber $\mathbf{W}$, et al. Orchestrating high-throughput genomic analysis with bioconductor. Nat Methods. 2015;12:115-21.

66. Mermel, C.H. et al. GISTIC2.0 facilitates sensitive and confident localization of the targets of focal somatic copy-number alteration in human cancers. Genome Biol 12, R41 (2011).

67. Trapnell C, Pachter L, Salzberg SL. TopHat: discovering splice junctions with RNA-Seq. Bioinformatics. 2009;25:1105-11.

68. Wang L, Wang S, Li W. RSeQC: quality control of RNA-seq experiments. Bioinformatics. 2012:28:2184-5.

69. Anders S, Pyl PT, Huber W. HTSeq--a Python framework to work with highthroughput sequencing data. Bioinformatics. 2015;31:166-9.

70. Li B, Dewey CN. RSEM: accurate transcript quantification from RNA-Seq data with or without a reference genome. BMC Bioinformatics. 2011;12:323.

71. Barbie DA, et al. Systematic RNA interference reveals that oncogenic KRASdriven cancers require TBK1. Nature. 2009:462:108-12.

72. Subramanian A, et al. Gene set enrichment analysis: a knowledge-based approach for interpreting genome-wide expression profiles. Proc Natl Acad Sci U S A. 2005;102:15545-50

73. Liberzon A, et al. Molecular signatures database (MSigDB) 3.0. Bioinformatics. 2011;27:1739-40

74. Law, C.W., Chen, Y., Shi, W. \& Smyth, G.K. voom: Precision weights unlock linear model analysis tools for RNA-seq read counts. Genome Biol 15, R29 (2014).

75. Cerami $\mathrm{E}$, et al. The cBio cancer genomics portal: an open platform for exploring multidimensional cancer genomics data. Cancer Discov. 2012;2: 401-4.

76. Aran D, Hu Z, Butte AJ. xCell: digitally portraying the tissue cellular heterogeneity landscape. Genome Biol. 2017;18:220.

77. Pruitt KD, Tatusova T, Maglott DR. NCBI reference sequence (RefSeq): a curated non-redundant sequence database of genomes, transcripts and proteins. Nucleic Acids Res. 2005;33:D501-4.

78. Shukla SA, et al. Comprehensive analysis of cancer-associated somatic mutations in class I HLA genes. Nat Biotechnol. 2015;33:1152-8.
79. Warren RL, et al. Derivation of HLA types from shotgun sequence datasets. Genome Med. 2012:4:95.

80. Andreatta $\mathrm{M}$, et al. Accurate pan-specific prediction of peptide-MHC class ॥ binding affinity with improved binding core identification. Immunogenetics. 2015:67:641-50

81. Hoof I, et al. NetMHCpan, a method for MHC class I binding prediction beyond humans. Immunogenetics. 2009;61:1-13.

82. Nielsen, M. \& Andreatta, M. NetMHCpan-3.0; improved prediction of binding to $\mathrm{MHC}$ class I molecules integrating information from multiple receptor and peptide length datasets. Genome Med 8, 33 (2016).

83. Yadav $\mathrm{M}$, et al. Predicting immunogenic tumour mutations by combining mass spectrometry and exome sequencing. Nature. 2014;515:572-6.

84. Odunsi $K$, et al. Efficacy of vaccination with recombinant vaccinia and fowlpox vectors expressing NY-ESO-1 antigen in ovarian cancer and melanoma patients. Proc Natl Acad Sci U S A. 2012;109:5797-802.

85. Atanackovic D, et al. Monitoring CD4+ T cell responses against viral and tumor antigens using T cells as novel target APC. J Immunol Methods. 2003; 278:57-66.

86. Tsuji T, et al. Antibody-targeted NY-ESO-1 to mannose receptor or DEC-205 in vitro elicits dual human CD8+ and CD4+ T cell responses with broad antigen specificity. J Immunol. 2011;186:1218-27.

87. Tsuji T, Altorki NK, Ritter G, Old LJ, Gnjatic S. Characterization of preexisting MAGE-A3-specific CD4+ T cells in cancer patients and healthy individuals and their activation by protein vaccination. J Immunol. 2009;183:4800-8.

88. Matsuzaki J, et al. Nonclassical antigen-processing pathways are required for MHC class II-restricted direct tumor recognition by NY-ESO-1-specific CD4(+) T cells. Cancer Immunol Res. 2014;2:341-50.

89. Matsuzaki J, et al. Direct tumor recognition by a human CD4(+) T-cell subset potently mediates tumor growth inhibition and orchestrates antitumor immune responses. Sci Rep. 2015;5:14896.

\section{Publisher's Note}

Springer Nature remains neutral with regard to jurisdictional claims in published maps and institutional affiliations.

Ready to submit your research? Choose BMC and benefit from:

- fast, convenient online submission

- thorough peer review by experienced researchers in your field

- rapid publication on acceptance

- support for research data, including large and complex data types

- gold Open Access which fosters wider collaboration and increased citations

- maximum visibility for your research: over $100 \mathrm{M}$ website views per year

At $\mathrm{BMC}$, research is always in progress.

Learn more biomedcentral.com/submissions 